\title{
Pushing Radical Cyclization from Regioselective to Regiospecific: Cyclization of Amidyl Radicals Controlled by Vinylic Halogen Substitution
}

\author{
Tianshun Hu, Meihua Shen, Qian Chen, and Chaozhong Li* \\ Shanghai Institute of Organic Chemistry, Chinese Academy of Sciences, 354 Fenglin \\ Road, Shanghai 200032, P. R. China \\ clig@mail.sioc.ac.cn
}

\section{Supporting Information}

\section{List of Contents}

1. Table S1. The results of iodolactonization

$\mathrm{S} 2-\mathrm{S} 3$

2. Typical procedures for amidyl radical cyclization.

$\mathrm{S} 3-\mathrm{S} 4$

3. Characterizations of compounds 1-21.

S4 - S16

4. Computational results on radicals 22 and 23.

S16 - S25

5. ${ }^{1} \mathrm{H}$ or ${ }^{13} \mathrm{C}$ NMR spectra of compounds without elemental analysis.

S26 - S45 
$\mathrm{Pb}(\mathrm{OAc})_{4}(96 \%)$ was purchased from Aldrich. $\mathrm{CH}_{2} \mathrm{Cl}_{2}$ was refluxed with $\mathrm{CaH}_{2}$ and distilled prior to use. All products were isolated by column chromatography on silica gel with hexane - acetone in an appropriate ratio as the eluent. ${ }^{1} \mathrm{H}$ NMR and ${ }^{13} \mathrm{C}$ NMR spectra were recorded on a $300 \mathrm{MHz} \mathrm{NMR}$ spectrometer with TMS as the internal standard. The conditions for ordinary HPLC analysis: Diamonsil-C18 column, size: $4.6 \times 150 \mathrm{~mm}$, UV detector $(220 \mathrm{~nm})$, mobile phase: acetonitrile/water, $60 / 40$ to $100 / 0$.

Table S1. The results of iodolactonization

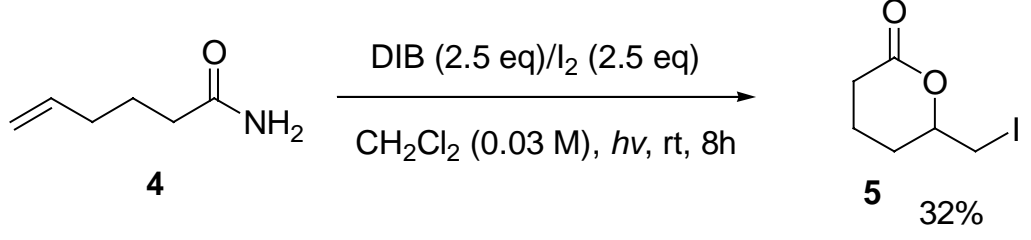

$$
\underset{\mathrm{CH}_{2} \mathrm{Cl}_{2}(0.03 \mathrm{M}), h v, \mathrm{rt}, 4 \mathrm{~h}}{\stackrel{\mathrm{Pb}(\mathrm{OAc})_{4}(3 \mathrm{eq}) / \mathrm{I}_{2}(2.5 \mathrm{eq})}{\longrightarrow}} \quad 14 \%
$$

$$
\frac{\operatorname{AgOAc}(2.5 \mathrm{eq}) / /_{2}(2.5 \mathrm{eq}) / \mathrm{BuOH}(3 \mathrm{eq})}{\mathrm{CH}_{2} \mathrm{Cl}_{2}(0.03 \mathrm{M}), h v, \mathrm{rt}, 4 \mathrm{~h}} \quad 15 \%
$$<smiles>C=CCCC(C)(C)C(N)=O</smiles>

$\operatorname{AgOAc}(2.5 \mathrm{eq}) / /_{2}(2.5 \mathrm{eq}) / \mathrm{t} \mathrm{BuOH}(3 \mathrm{eq})$

$\mathrm{CH}_{2} \mathrm{Cl}_{2}(0.03 \mathrm{M}), h v, \mathrm{rt}, 4 \mathrm{~h}$

$\mathrm{CH}_{2} \mathrm{Cl}_{2}(0.03 \mathrm{M}), h v, \mathrm{rt}, 4 \mathrm{~h}$<smiles>CC=CCCCC(N)=O</smiles>

$\mathrm{Pb}(\mathrm{OAc})_{4}(3 \mathrm{eq}) / /_{2}(2.5 \mathrm{eq})$

$\mathrm{CH}_{2} \mathrm{Cl}_{2}(0.03 \mathrm{M}), h v, \mathrm{rt}, 4 \mathrm{~h}$

$\mathrm{K}_{2} \mathrm{CO}_{3}(2 \mathrm{eq}) / /_{2}(3 \mathrm{eq})$

$\mathrm{CH}_{2} \mathrm{Cl}_{2}(0.03 \mathrm{M}), h v$, rt, $4 \mathrm{~h}$

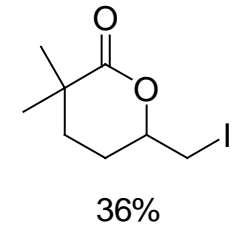

$43 \%$<smiles>CC(I)C1CCCC(=O)O1</smiles>

$39 \%$ 
<smiles>O=C1CCCC(C(I)I)O1</smiles>

This compound was isolated from the dark reaction of $6 \mathbf{a}$ with $\mathrm{Pb}(\mathrm{OAc})_{4} / \mathrm{I}_{2}$ at $\mathrm{rt}$ (see manuscript) as a yellowish oil. Yield: $37 \% .{ }^{1} \mathrm{H}$ NMR $\left(300 \mathrm{MHz}, \mathrm{CDCl}_{3}\right) \delta 1.62-1.71(1 \mathrm{H}, \mathrm{m}), 1.83-1.96(2 \mathrm{H}, \mathrm{m}), 2.36-$ $2.48(2 \mathrm{H}, \mathrm{m}), 2.53-2.62(1 \mathrm{H}, \mathrm{m}), 3.85(1 \mathrm{H}, \mathrm{dt}, J=10.5,3.6 \mathrm{~Hz}), 5.21(1 \mathrm{H}, \mathrm{d}, J=3.6 \mathrm{~Hz}) .{ }^{13} \mathrm{C} \mathrm{NMR}$ $\left(\mathrm{CDCl}_{3}\right) \delta-23.4,17.8,27.5,29.3,82.7,176.8$. EIMS: $m / z$ (rel intensity) $366\left(\mathrm{M}^{+}, 21\right), 239(32), 211$ (100), 169 (37), 141 (12), 127 (9), 99 (16), 55 (31). HRMS calcd for $\mathrm{C}_{6} \mathrm{H}_{8} \mathrm{I}_{2} \mathrm{O}_{2}\left(\mathrm{M}^{+}\right)$: 365.8614. Found: 365.8617 .<smiles>O=C1CCCC(CI)=N1</smiles>

This compound was isolated in $37 \%$ yield from the reaction of $\mathbf{8 c}$ with $\mathrm{NaHCO}_{3} / \mathrm{I}_{2}$ in acetonitrile at rt for $16 \mathrm{~h}$ (see manuscript). Yellowish oil. ${ }^{1} \mathrm{H}$ NMR $\left(300 \mathrm{MHz}, \mathrm{CDCl}_{3}\right) \delta 1.94-2.04(2 \mathrm{H}, \mathrm{m}), 2.45(2 \mathrm{H}, \mathrm{t}$, $J=6.9 \mathrm{~Hz}), 2.95(2 \mathrm{H}, \mathrm{t}, J=6.8 \mathrm{~Hz}), 3.84(2 \mathrm{H}, \mathrm{d}, J=0.9 \mathrm{~Hz}) ;{ }^{13} \mathrm{C} \mathrm{NMR}\left(\mathrm{CDCl}_{3}\right) \delta 5.6,16.1,19.6,36.8$, 119.0, 201.4; EIMS m/z (rel intensity) $237\left(\mathrm{M}^{+}, 12\right), 209$ (4), 195 (3), 169 (17), 141 (11), 110 (16), 96 (100), 68 (48); Anal. calcd for $\mathrm{C}_{6} \mathrm{H}_{8} \mathrm{INO}$. C, 30.40; H, 3.40; N, 5.91. Found: C, 30.42; H, 3.52; N, 5.83.

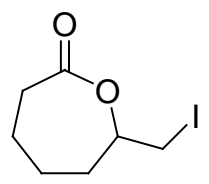

Colorless oil. Yield: $25 \%$. ${ }^{1} \mathrm{H}$ NMR $\left(300 \mathrm{MHz}, \mathrm{CDCl}_{3}\right) \delta 1.62-1.73(2 \mathrm{H}, \mathrm{m}), 1.94-2.02(2 \mathrm{H}, \mathrm{m}), 2.13-$ $2.23(2 \mathrm{H}, \mathrm{m}), 2.53-2.63(1 \mathrm{H}, \mathrm{m}), 2.68-2.76(1 \mathrm{H}, \mathrm{m}), 3.25-3.39(2 \mathrm{H}, \mathrm{m}), 4.30-4.37(1 \mathrm{H}, \mathrm{m})$.

Known compound: Simonot, B.; Rousseau, G. J. Org. Chem. 1994, 59, 5912.

Typical Procedure for Bu, $\mathrm{SnH}$-Initiated Amidyl Radical Cyclization: $N$-(Phenylthio)hept-5enamide (1) (235 mg, $1.0 \mathrm{mmol}$ ) was dissolved in $80 \mathrm{~mL}$ of anhydrous benzene, and the solution was brought to reflux. Into this refluxing solution was added with the aid of a syringe pump over a period of $4 \mathrm{~h} \mathrm{Bu} 3 \mathrm{SnH}(0.54 \mathrm{~mL}, 2 \mathrm{mmol})$ and $\mathrm{AIBN}(49 \mathrm{mg}, 0.3 \mathrm{mmol})$ in benzene solution $(20 \mathrm{~mL})$ under nitrogen atmosphere. The mixture was cooled down to room temperature and checked by HPLC. After removal of the solvent, the residue was chromatographed on silica gel with hexane-acetone (100:1 to 5:1) as the eluent to give lactam 2 (85.1 mg, 67\% yield), lactam 3 (10.2 mg, 8\% yield) and hept-5enamide (19.1 mg, 15\%).

\section{Typical Procedures for $\mathrm{Pb}(\mathrm{OAc})_{4} / \mathrm{I}_{2}$-Initiated Amidyl Radical Cyclization.}

Method A: To the solution of $\mathrm{Pb}(\mathrm{OAc})_{4}(466 \mathrm{mg}, 1.05 \mathrm{mmol})$ in dry $\mathrm{CH}_{2} \mathrm{Cl}_{2}(10 \mathrm{~mL})$ was added iodine $(190 \mathrm{mg}, 0.75 \mathrm{mmol})$ at $\mathrm{rt}$ under nitrogen atmosphere. The mixture was stirred for $3 \mathrm{~min}$, then a haloenamide $(0.3 \mathrm{mmol})$ was added and the resulting mixture was irradiated at $\mathrm{rt}$ with a $125 \mathrm{~W}$ high pressure mercury lamp for $4 \mathrm{~h}$. Aqueous $\mathrm{Na}_{2} \mathrm{~S}_{2} \mathrm{O}_{3}(10 \mathrm{~mL})$ was then added. The two layers were separated and the aqueous phase was extracted with $\mathrm{CH}_{2} \mathrm{Cl}_{2}(3 \times 10 \mathrm{~mL})$. The combined organic layer was washed with aqueous $\mathrm{Na}_{2} \mathrm{CO}_{3}$ and brine, dried over anhydrous $\mathrm{Na}_{2} \mathrm{SO}_{4}$. After removal of the solvent, the crude product was purified by column chromatography on silica gel using hexane-acetone $(4: 1)$ as the eluent to give the products. 
Method B: The same procedure in Method A was followed except that the reaction temperature was set at $0{ }^{\circ} \mathrm{C}$.

Method C: The same procedure in Method A was followed except that the reaction temperature was set at $0{ }^{\circ} \mathrm{C}$ and that the amount of iodine was 1.3 equiv. rather than 2.5 equiv.

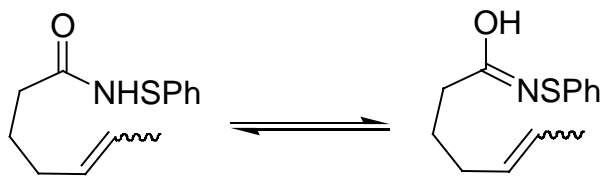

White solid. Yield: $48 \%$. Mp 44-45 ${ }^{\circ} \mathrm{C}$. Two isomers (2:1). ${ }^{1} \mathrm{H}$ NMR $\left(300 \mathrm{MHz}, \mathrm{CDCl}_{3}\right) \delta 1.56-1.76$ $(5 \mathrm{H}, \mathrm{m}), 2.01-2.16(2 \mathrm{H}, \mathrm{m}), 2.35-2.53(2 \mathrm{H}, \mathrm{m}), 5.36-5.46(2 \mathrm{H}, \mathrm{m}), 6.49$ and $6.87(1 \mathrm{H}, \mathrm{br}), 7.20-7.26$ $(5 \mathrm{H}, \mathrm{m}) .{ }^{13} \mathrm{C} \mathrm{NMR}\left(\mathrm{CDCl}_{3}\right) \delta 12.8 / 17.8,24.4 / 25.3,26.1 / 30.3,31.9 / 35.9,117.9 / 123.3,125.1 / 125.6$, 126.1/126.8, 129.0/129.2, 130.0, 138.8, 174.9/180.0. EIMS: $\mathrm{m} / \mathrm{z}$ (rel intensity) $236\left(\mathrm{M}^{+}+1,5\right), 202$ (2), 167 (3), 150 (10), 125 (100), 109 (28), 93 (23), 55 (75). Anal. calcd for $\mathrm{C}_{13} \mathrm{H}_{17} \mathrm{NOS}$ : C, 66.34; H, 7.28; N, 5.95. Found: C, 66.45; H, 7.25; N, 5.69.<smiles>C=C(C)CCCC(O)=NSc1ccccc1</smiles>

Colorless oil. Yield: 51\%. Two isomers (2.3:1). ${ }^{1} \mathrm{H}$ NMR $\left(300 \mathrm{MHz}, \mathrm{CDCl}_{3}\right) \delta 1.69(3 \mathrm{H}, \mathrm{s}), 1.74-1.88$ $(2 \mathrm{H}, \mathrm{m}), 2.01-2.06(2 \mathrm{H}, \mathrm{m}), 2.35 / 2.53(2 \mathrm{H}, \mathrm{m}), 4.66-4.73(2 \mathrm{H}, \mathrm{m}), 6.59 / 7.11(1 \mathrm{H}, \mathrm{br}), 7.18-7.35(5 \mathrm{H}$, $\mathrm{m}) .{ }^{13} \mathrm{C} \mathrm{NMR}\left(\mathrm{CDCl}_{3}\right) \delta 22.0 / 22.3,23.2,30.2,35.6 / 36.8,110.5,123.8,124.6,126.5,128.7,129.1$, 138.8, 144.6, 175.7. EIMS: $m / z$ (rel intensity) $235\left(\mathrm{M}^{+}, 4\right), 218$ (1), 150 (2), 150 (3), 125 (100), 109 (15), 93 (18), 55 (66). Anal. calcd for $\mathrm{C}_{13} \mathrm{H}_{17} \mathrm{NOS}$ : C, 66.34; H, 7.28; N, 5.95. Found: C, 66.27; H, $7.45 ; \mathrm{N}, 5.81$.
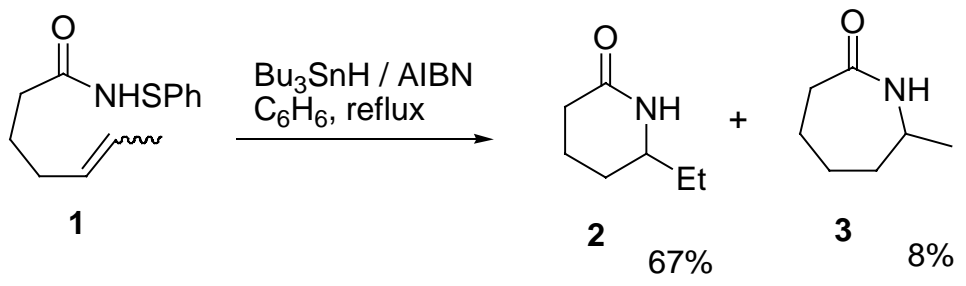

$(8: 1)$<smiles>C=C(C)CCCC(=O)N[SnH2]</smiles>

A

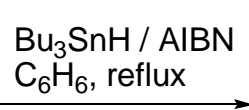<smiles>CC1CCCC(=O)NC1</smiles>

$41 \%$<smiles>CC1(C)CCCC(=O)N1</smiles>

$3 \%$

(14:1)<smiles>NC(=O)CCC/C=C/Br</smiles> 
White solid. Yield: 70\%. $Z: E=55: 45$ (HPLC). ${ }^{1} \mathrm{H}$ NMR $\left(300 \mathrm{MHz}, \mathrm{CDCl}_{3}\right) \delta$ 1.71-1.84 (2H, m), 2.09-2.31 (4H, m), $5.61(1 \mathrm{H}, \mathrm{br}), 5.95(1 \mathrm{H}, \mathrm{br}), 6.04-6.23(2 \mathrm{H}, \mathrm{m}) .{ }^{13} \mathrm{C} \mathrm{NMR}\left(\mathrm{CDCl}_{3}\right) \delta 23.7 / 24.0$, 29.0/32.1, 34.6/34.9, 105.1/108.7, 133.7/136.9, 175.5. EIMS $m / z$ (rel intensity) $191\left(\mathrm{M}^{+}, 2\right), 155$ (2), 119 (8), 112 (93), 98 (8), 85 (6), 59 (100), 44 (41). Anal. calcd for $\mathrm{C}_{6} \mathrm{H}_{10}$ BrNO: C, 37.52; H, 5.25; N, 7.29. Found: C, 37.27; H, 5.18; N, 7.08.

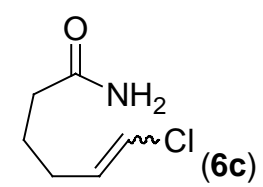

White solid. Yield: 65\%. $Z: E=14: 86$ (HPLC). ${ }^{1} \mathrm{H}$ NMR (300 MHz, $\left.\mathrm{CDCl}_{3}\right) \delta$ 1.70-1.80 (2H, m), 2.10-2.33 (2H, m), $5.54(1 \mathrm{H}, \mathrm{br}), 5.75-6.08(3 \mathrm{H}, \mathrm{m}) .{ }^{13} \mathrm{C} \mathrm{NMR}\left(\mathrm{CDCl}_{3}\right) \delta 24.0 / 24.3,26.2 / 30.1$, 34.6/34.9, 117.7/117.9, 130.5/132.8, 174.9. EIMS $m / z$ (rel intensity) $148\left(\mathrm{M}^{+}+1,21\right), 112$ (11), 100 (1), 95 (2), 75 (9), 67 (8), 59 (100), 44 (49). Anal. calcd for $\mathrm{C}_{6} \mathrm{H}_{10} \mathrm{ClNO}$ : C, 48.82; H, 6.83; N, 9.49. Found: C, 49.00; H, 6.81; N, 9.59.

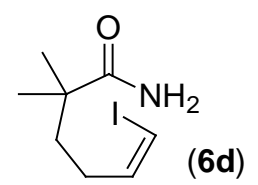

Yellowish solid. Yield: 60\%. Mp 74-76 ${ }^{\circ} \mathrm{C} .{ }^{1} \mathrm{H}$ NMR $\left(300 \mathrm{MHz}, \mathrm{CDCl}_{3}\right) \delta 1.25(6 \mathrm{H}, \mathrm{s}), 1.61-1.66(2 \mathrm{H}$, $\mathrm{m}), 2.11-2.19(2 \mathrm{H}, \mathrm{m}), 5.64(2 \mathrm{H}, \mathrm{br}), 6.16-6.19(2 \mathrm{H}, \mathrm{m}) .{ }^{13} \mathrm{C} \mathrm{NMR}\left(\mathrm{CDCl}_{3}\right) \delta 25.5,30.6,38.9,41.9$, 82.7, 140.6, 179.9. EIMS: $m / z$ (rel intensity) $268\left(\mathrm{M}^{+}+1,1\right), 181$ (2), 167 (8), 140 (3), 127 (1), 87 (100), 72 (19), 41 (15). Anal. calcd for $\mathrm{C}_{8} \mathrm{H}_{14} \mathrm{INO}$ : C, 35.97; H, 5.28; N, 5.24. Found: C, 36.13; H, 5.08; N, 5.31 .

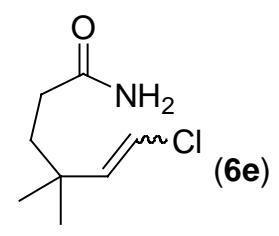

Yellowish solid. Yield: $72 \%$. Mp 46-48 ${ }^{\circ} \mathrm{C} . Z: E=30: 70 .{ }^{1} \mathrm{H}$ NMR $\left(300 \mathrm{MHz}, \mathrm{CDCl}_{3}\right) \delta 1.21(6 \mathrm{H}, \mathrm{s})$, 1.88-1.92 (2H, m), 2.12-2.24 (2H, m), $5.48(2 \mathrm{H}, \mathrm{br}), 5.55-5.58(1 \mathrm{H}, \mathrm{m}), 5.96-5.99(1 \mathrm{H}, \mathrm{m}),{ }^{13} \mathrm{C} \mathrm{NMR}$ $\left(\mathrm{CDCl}_{3}\right) \delta 26.7 / 27.7,31.3 / 31.7,36.3,37.5 / 37.7,116.1 / 116.9,138.2 / 142.3,175.7$. EIMS: $\mathrm{m} / z$ (rel intensity) $176\left(\mathrm{M}^{+}+1,24\right), 140$ (68), 123 (15), 103 (31), 95 (43), 81 (35), 67 (66), 44 (100). HRMS calcd for $\mathrm{C}_{8} \mathrm{H}_{14} \mathrm{NO}\left(\mathrm{M}^{+}-\mathrm{Cl}\right): 140.1075$. Found: 140.1071 .

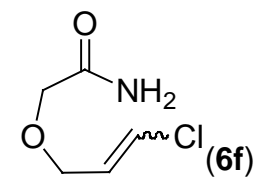

Yellowish oil. Yield: 66\%. $Z: E=40: 60 .{ }^{1} \mathrm{H}$ NMR (300MHz, $\left.\mathrm{CDCl}_{3}\right) \delta 3.94-3.97(2 \mathrm{H}, \mathrm{m}), 4.06(1 \mathrm{H}$, dd, $J=6.6,1.5 \mathrm{~Hz}), 4.29(1 \mathrm{H}, \mathrm{dd}, J=6.6,1.5 \mathrm{~Hz}), 5.92-6.07(1 \mathrm{H}, \mathrm{m}), 6.24-6.33(1 \mathrm{H}, \mathrm{m}), 6.56(2 \mathrm{H}, \mathrm{br})$. ${ }^{13} \mathrm{C} \mathrm{NMR}\left(\mathrm{CDCl}_{3}\right) \delta 66.3,69.1 / 69.3,121.7 / 122.4,127.1 / 128.7,172.4 / 172.6$. EIMS: $\mathrm{m} / z$ (rel intensity) $150\left(\mathrm{M}^{+}+1,17\right), 132$ (1), 114 (37), 89 (3), 105 (2), 75 (79), 59 (100), 44 (70). Anal. calcd for $\mathrm{C}_{5} \mathrm{H}_{8} \mathrm{ClNO}_{2}$ : C, 40.15; H, 5.39; N, 9.36. Found: C, 40.18; H, 5.38; N, 9.30. 
<smiles>O=C1CCCC(C(I)I)N1</smiles>

Method A: White solid. Yield: 85\%. Mp 160-161 ${ }^{\circ} \mathrm{C} .{ }^{1} \mathrm{H}$ NMR $\left(300 \mathrm{MHz}, \mathrm{CDCl}_{3}\right) \delta 1.39-1.53(1 \mathrm{H}, \mathrm{m})$, 1.72-1.87 $(1 \mathrm{H}, \mathrm{m}), 1.91-2.00(1 \mathrm{H}, \mathrm{m}), 2.17-2.48(3 \mathrm{H}, \mathrm{m}), 3.19-3.24(1 \mathrm{H}, \mathrm{m}), 5.16(1 \mathrm{H}, \mathrm{d}, J=3.8 \mathrm{~Hz})$, $6.44(1 \mathrm{H}, \mathrm{br}) .{ }^{13} \mathrm{C}$ NMR $\left(\mathrm{CDCl}_{3}\right) \delta-14.9,18.8,28.9,31.1,60.5,172.8$. EIMS $\mathrm{m} / z$ (rel intensity) 366 $\left(\mathrm{M}^{+}+1,1\right), 365\left(\mathrm{M}^{+}, 2\right), 238$ (2), 167 (3), 127 (5), 111 (6), 98 (100), 82 (9), 55 (25). HRMS calcd for $\mathrm{C}_{6} \mathrm{H}_{9} \mathrm{I}_{2} \mathrm{NO}\left(\mathrm{M}^{+}+\mathrm{H}\right)$ 365.8846. Found 365.8862.<smiles>O=C1CCCC(C(Br)I)N1</smiles>

Method A: White solid. Two stereoisomers in $\sim 1: 1$ ratio. Yield: $79 \%$. ${ }^{1} \mathrm{H}$ NMR $\left(300 \mathrm{MHz}, \mathrm{CDCl}_{3}\right)$ 1.43-1.58 $(1 \mathrm{H}, \mathrm{m}), 1.61-1.79(1 \mathrm{H}, \mathrm{m}), 1.81-1.96(1 \mathrm{H}, \mathrm{m}), 2.03-2.41(3 \mathrm{H}, \mathrm{m}), 3.34-3.48(1 \mathrm{H}, \mathrm{m}), 5.49$ $(1 \mathrm{H}, \mathrm{m}), 6.64 / 6.78(1 \mathrm{H}, \mathrm{br}) .{ }^{13} \mathrm{C} \mathrm{NMR}\left(\mathrm{CDCl}_{3}\right) \delta 18.8 / 18.9,19.2 / 19.3,27.6 / 27.7,31.1 / 31.2,60.2 / 60.5$, 172.7. EIMS $m / z$ (rel intensity) $318\left(\mathrm{M}^{+}+1,3\right), 190$ (1), 127 (2), 121 (1), 98 (100), 82 (4), 55 (36). Anal. calcd. for $\mathrm{C}_{6} \mathrm{H}_{9} \mathrm{BrINO}$ : C, 22.67; H, 2.85; N, 4.41. Found: C, 22.70; H, 2.85; N, 4.38.<smiles>O=C1CCCC(C(Cl)I)N1</smiles>

Method A: White solid. Two stereoisomers in $\sim 1: 1$ ratio. Yield: $81 \% .{ }^{1} \mathrm{H}$ NMR $\left(300 \mathrm{MHz}, \mathrm{CDCl}_{3}\right) \delta$ 1.53-1.87 $(2 \mathrm{H}, \mathrm{m}), 1.92-2.03(1 \mathrm{H}, \mathrm{m}), 2.07-2.50(3 \mathrm{H}, \mathrm{m}), 3.39-3.45 / 3.61-3.67(1 \mathrm{H}, \mathrm{m}), 5.68 / 5.72(1 \mathrm{H}$, $\mathrm{d}, J=4.2 \mathrm{~Hz}), 6.44 / 6.58(1 \mathrm{H}, \mathrm{br}) .{ }^{13} \mathrm{C} \mathrm{NMR}\left(\mathrm{CDCl}_{3}\right) \delta 18.8 / 18.9,26.4 / 26.9,31.1 / 31.2,35.7 / 35.9$, 60.3/60.6, 172.7. EIMS $m / z$ (rel intensity) $274\left(\mathrm{M}^{+}+1,1\right), 175$ (1), 146 (1), 127 (5), 98 (100), 82 (4), 70 (7), 55 (76). Anal. calcd. for $\mathrm{C}_{6} \mathrm{H}_{9}$ CIINO: C, 26.35; H, 3.32; N, 5.12. Found: C, 26.69; H, 3.39; N, 5.18.<smiles>CC1(C)CCC(C(I)I)NC1=O</smiles>

Method C: White solid. Yield: $81 \%$. Mp 145-147 ${ }^{\circ} \mathrm{C} .{ }^{1} \mathrm{H}$ NMR $\left(300 \mathrm{MHz}, \mathrm{CDCl}_{3}\right) \delta 1.23(3 \mathrm{H}, \mathrm{s}), 1.29$ $(3 \mathrm{H}, \mathrm{s}), 1.60-1.85(4 \mathrm{H}, \mathrm{m}), 3.13-3.19(2 \mathrm{H}, \mathrm{m}), 5.17(1 \mathrm{H}, \mathrm{d}, J=3.3 \mathrm{~Hz}), 6.30(1 \mathrm{H}, \mathrm{br}) .{ }^{13} \mathrm{C} \mathrm{NMR}$ $\left(\mathrm{CDCl}_{3}\right) \delta-15.8,26.2,26.6,27.0,33.7,37.3,61.3,178.4$. EIMS: $m / z$ (rel intensity) $393\left(\mathrm{M}^{+}, 9\right), 254$ (1), 167 (4), 139 (3), 126 (100), 111 (5), 96 (21), 81 (41). HRMS calcd for $\mathrm{C}_{8} \mathrm{H}_{14} \mathrm{I}_{2} \mathrm{NO}\left(\mathrm{M}^{+}+1\right)$ : 393.9165. Found: 393.9155.<smiles></smiles>

Method B: White solid. Yield: $89 \%$. Mp $96-98{ }^{\circ} \mathrm{C}$. Two stereoisomers in $~ 5: 1$ ratio. ${ }^{1} \mathrm{H}$ NMR $(300 \mathrm{MHz}$, $\left.\mathrm{CDCl}_{3}\right) \delta 1.14(6 \mathrm{H}, \mathrm{s}), 1.42-1.51(1 \mathrm{H}, \mathrm{m}), 1.98-2.08(1 \mathrm{H}, \mathrm{m}), 2.36-2.48(2 \mathrm{H}, \mathrm{m}), 3.12 / 3.78(1 \mathrm{H}, 2 \mathrm{~d}, \mathrm{~J}=$ 
$2.7 \mathrm{~Hz}), 6.01 / 6.27(1 \mathrm{H}, 2 \mathrm{~d}, \mathrm{~J}=1.5 \mathrm{~Hz}) 6.24(1 \mathrm{H}, \mathrm{br}) .{ }^{13} \mathrm{C} \mathrm{NMR}\left(\mathrm{CDCl}_{3}\right) \delta$ 23.7/24.3, 27.9/28.1, 29.3/31.2, 32.2/32.4, 34.3, 68.6/70.5, 172.0. EIMS: $m / z$ (rel intensity) $301\left(\mathrm{M}^{+}, 1\right), 204$ (1), 174 (15), 126 (100), 104 (9), 84 (6), 69 (34), 55 (57). Anal. calcd for $\mathrm{C}_{8} \mathrm{H}_{13}$ ClINO: C, 31.86; H, 4.35; N, 4.64. Found: C, 32.09; H, 4.32; N, 4.47.<smiles>O=C1COCC(C(Cl)I)N1</smiles>

Method B: White solid. Yield: $81 \%$. Mp 131-133 ${ }^{\circ} \mathrm{C}$. Two stereoisomers in $\sim 1: 1$ ratio. ${ }^{1} \mathrm{H}$ NMR $\left(300 \mathrm{MHz}, \mathrm{CDCl}_{3}\right) \delta 3.73-3.92(2 \mathrm{H}, \mathrm{m}), 4.08(1 \mathrm{H}, \mathrm{d}, J=4.2 \mathrm{~Hz}), 4.11-4.18(2 \mathrm{H}, \mathrm{m}), 5.74 / 5.77(1 \mathrm{H}, 2 \mathrm{~d}$, $J=1.8 \mathrm{~Hz}), 7.18(1 \mathrm{H}, \mathrm{br}) .{ }^{13} \mathrm{C} \mathrm{NMR}\left(\mathrm{CDCl}_{3}\right) \delta 29.9 / 30.7,59.4 / 59.6,64.5 / 66.6,68.1 / 68.2,168.3 / 168.4$. EIMS: $m / z$ (rel intensity) $275\left(\mathrm{M}^{+}, 1\right), 183$ (6), 164 (4), 148 (5), 127 (5), 100 (100), 86 (31), 55 (10). Anal. calcd for $\mathrm{C}_{5} \mathrm{H}_{7} \mathrm{ClINO}_{2}$ : C, 21.80; H, 2.56; N, 5.08. Found: C, 21.94; H, 2.98; N, 5.01.

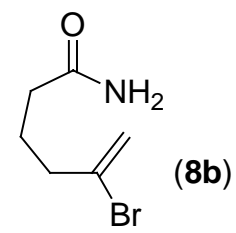

White solid. Yield: $76 \%$. Mp 70-72 ${ }^{\circ} \mathrm{C} .{ }^{1} \mathrm{H}$ NMR (300 MHz, $\left.\mathrm{CDCl}_{3}\right) \delta$ 1.85-1.94 (2H, m), 2.20-2.25 $(2 \mathrm{H}, \mathrm{m}), 2.48(2 \mathrm{H}, \mathrm{t}, J=7.0 \mathrm{~Hz}), 5.42(1 \mathrm{H}, \mathrm{s}), 5.60(1 \mathrm{H}, \mathrm{s}), 5.68(1 \mathrm{H}, \mathrm{br}), 6.17(1 \mathrm{H}, \mathrm{br}) ;{ }^{13} \mathrm{C} \mathrm{NMR}$ $\left(\mathrm{CDCl}_{3}\right) \delta 23.3,33.9,40.3,117.5,133.4,175.0$; EIMS $\mathrm{m} / z$ (rel intensity) $192\left(\mathrm{M}^{+}+1,17\right), 175(1), 119$ (1), 113 (8), 112 (100), 95 (6), 69 (34). Anal. calcd. for $\mathrm{C}_{6} \mathrm{H}_{10} \mathrm{BrNO}$ : C, 37.52; H, 5.25; N, 7.29. Found: C, 37.44; H, 5.31; N, 7.24.

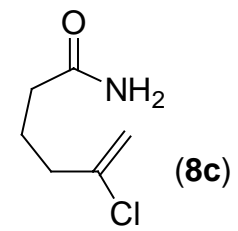

White solid. Yield: 69\%. Mp 72-74 ${ }^{\circ} \mathrm{C} .{ }^{1} \mathrm{H}$ NMR (300 MHz, $\left.\mathrm{CDCl}_{3}\right) \delta 1.80-1.89(2 \mathrm{H}, \mathrm{m}), 2.16-2.21$ $(2 \mathrm{H}, \mathrm{m}), 2.34(2 \mathrm{H}, \mathrm{t}, J=7.2 \mathrm{~Hz}), 5.11-5.13(2 \mathrm{H}, \mathrm{m}), 5.93(1 \mathrm{H}, \mathrm{br}), 6.48(1 \mathrm{H}, \mathrm{br}) .{ }^{13} \mathrm{C} \mathrm{NMR}\left(\mathrm{CDCl}_{3}\right) \delta$ 22.6, 34.0, 38.1, 112.8, 141.6, 175.4. EIMS $m / z$ (rel intensity) $148\left(\mathrm{M}^{+}+1,1\right), 113$ (7), 112 (100), 95 (2), 69 (16), 67 (10), 59 (95), 44 (41); Anal. calcd. for $\mathrm{C}_{6} \mathrm{H}_{10} \mathrm{ClNO}$ : C, 48.82; H, 6.83; N, 9.49. Found: C, 48.70; H, 6.81; N, 9.34.

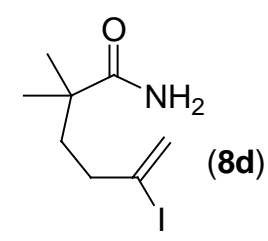

Reddish solid. Yield: 55\%. Mp 76-78 ${ }^{\circ} \mathrm{C} .{ }^{1} \mathrm{H}$ NMR (300MHz, $\left.\mathrm{CDCl}_{3}\right) \delta 1.26(6 \mathrm{H}, \mathrm{s}), 1.72-1.79(2 \mathrm{H}, \mathrm{m})$, 2.38-2.43 $(2 \mathrm{H}, \mathrm{m}), 5.45(1 \mathrm{H}, \mathrm{br}), 5.63(1 \mathrm{H}, \mathrm{br}), 5.68-5.70(1 \mathrm{H}, \mathrm{m}), 6.04-6.05(1 \mathrm{H}, \mathrm{m}) .{ }^{13} \mathrm{C} \mathrm{NMR}$ $\left(\mathrm{CDCl}_{3}\right) \delta 25.6,40.8,41.1,41.5,111.4,125.5,179.6$. EIMS: $m / z$ (rel intensity) $268\left(\mathrm{M}^{+}+1,4\right), 181$ (2), 167 (5), 140 (100), 127 (4), 112 (5), 87 (38), 55 (72). Anal. calcd for $\mathrm{C}_{8} \mathrm{H}_{14} \mathrm{INO}$ : C, 35.97; H, 5.28; N, 5.24. Found: C, 36.24; H, 5.21; N, 5.23. 


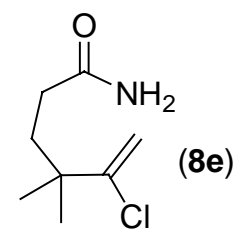

Yellowish solid. Yield: 66\%. Mp 71-73 ${ }^{\circ} \mathrm{C} .{ }^{1} \mathrm{H}$ NMR $\left(300 \mathrm{MHz}, \mathrm{CDCl}_{3}\right) \delta 1.19(6 \mathrm{H}, \mathrm{s}), 1.80-1.86(2 \mathrm{H}$, $\mathrm{m}), 2.08-2.17(2 \mathrm{H}, \mathrm{m}), 5.17(1 \mathrm{H}, \mathrm{t}, J=1.8 \mathrm{~Hz}), 5.24(1 \mathrm{H}, \mathrm{t}, J=1.8 \mathrm{~Hz}), 5.46(1 \mathrm{H}, \mathrm{br}), 5.63(1 \mathrm{H}, \mathrm{br})$. ${ }^{13} \mathrm{C}$ NMR $\left(\mathrm{CDCl}_{3}\right) \delta 26.6,31.3,35.6,41.4,111.9,150.4,175.4$. EIMS: $\mathrm{m} / z$ (rel intensity) $176\left(\mathrm{M}^{+}+1\right.$, 1), 140 (74), 123 (10), 114 (11), 95 (20), 81 (21), 67 (46), 44 (100). HRMS calcd for $\mathrm{C}_{8} \mathrm{H}_{14} \mathrm{NO}\left(\mathrm{M}^{+}-\right.$ Cl): 140.1075. Found: 140.1075.

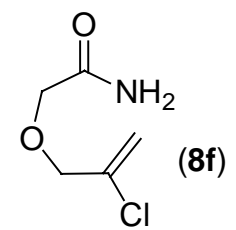

Yellowish solid. Yield: 67\%. Mp 68-70 ${ }^{\circ} \mathrm{C} .{ }^{1} \mathrm{H}$ NMR $\left(300 \mathrm{MHz}, \mathrm{CDCl}_{3}\right) \delta 4.00(2 \mathrm{H}, \mathrm{s}), 4.15(1 \mathrm{H}, \mathrm{s})$, $5.45(1 \mathrm{H}, \mathrm{d}, J=1.8 \mathrm{~Hz}), 5.48(1 \mathrm{H}, \mathrm{d}, J=1.2 \mathrm{~Hz}), 5.77(1 \mathrm{H}, \mathrm{br}), 6.57(1 \mathrm{H}, \mathrm{br}) .{ }^{13} \mathrm{C} \mathrm{NMR}\left(\mathrm{CDCl}_{3}\right) \delta$ 69.2, 73.9, 115.3, 137.1, 171.8. EIMS: $m / z$ (rel intensity) $149\left(\mathrm{M}^{+}, 1\right), 114(6), 105(2), 92(1), 75$ (24 ), 59 (100), 49 (8), 44 (62). Anal. calcd for $\mathrm{C}_{5} \mathrm{H}_{8} \mathrm{ClNO}_{2}$ : C, 40.15; H, 5.39; N, 9.36. Found: C, 40.17; $\mathrm{H}$, $5.31 ; \mathrm{N}, 9.29$.

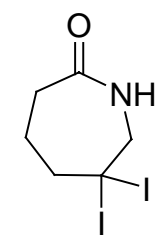

$(9 a)$

Method A: White solid. Yield: 72\%. Mp 136-138 ${ }^{\circ} \mathrm{C} .{ }^{1} \mathrm{H}$ NMR $\left(300 \mathrm{MHz}, \mathrm{CDCl}_{3}\right) \delta 1.61(2 \mathrm{H}$, br), 2.47-2.51 (2H, m), $2.77(2 \mathrm{H}, \mathrm{br}), 3.54(2 \mathrm{H}, \mathrm{d}, J=5.7 \mathrm{~Hz}), 6.57(1 \mathrm{H}, \mathrm{br}) .{ }^{13} \mathrm{C} \mathrm{NMR}\left(\mathrm{CDCl}_{3}\right) \delta 14.8$, 24.3, 35.4, 58.2, 61.0, 177.3. EIMS $m / z$ (rel intensity) $365\left(\mathrm{M}^{+}, 3\right), 280$ (1), 238 (39), 181 (8), 167 (7), 127 (12), 111 (100), 82 (35). HRMS calcd for $\mathrm{C}_{6} \mathrm{H}_{9} \mathrm{I}_{2} \mathrm{NO}\left(\mathrm{M}^{+}+\mathrm{H}\right)$ 365.8846. Found 365.8862.<smiles>O=C1CCCC(I)(I)CN1</smiles>

(9b)

Method A: White solid. Yield: 89\%. Mp 123-125 ${ }^{\circ} \mathrm{C} .{ }^{1} \mathrm{H}$ NMR $\left(300 \mathrm{MHz}, \mathrm{CDCl}_{3}\right) \delta 1.68-1.88(2 \mathrm{H}, \mathrm{m})$, 2.47-2.55 (2H, m), 2.59-2.67 (1H, m), 2.83-2.92 (1H, m), 3.56-3.59 $(1 \mathrm{H}, \mathrm{m}), 3.74-3.81(1 \mathrm{H}, \mathrm{m}), 6.70$ $\left(1 \mathrm{H}\right.$, br). ${ }^{13} \mathrm{C} \mathrm{NMR}\left(\mathrm{CDCl}_{3}\right) \delta 23.1,35.4,55.8,59.2,177.2$. EIMS $m / z$ (rel intensity) $318\left(\mathrm{M}^{+}+1,4\right)$, 289 (1), 190 (52), 133 (15), 110 (62), 82 (52), 67 (100). Anal. calcd. for $\mathrm{C}_{6} \mathrm{H}_{9} \mathrm{BrINO}$ : C, 22.67; H, $2.85 ; \mathrm{N}, 4.41$. Found: C, 22.94; H, 2.81; N, 4.24 .<smiles>O=C1CCCC(Cl)(I)CN1</smiles>

Method A: White solid. Yield: 70\%. Mp 139-141 ${ }^{\circ} \mathrm{C} .{ }^{1} \mathrm{H}$ NMR $\left(300 \mathrm{MHz}, \mathrm{CDCl}_{3}\right) \delta 1.66-1.69(1 \mathrm{H}, \mathrm{m})$, 1.87 -1.95 $(1 \mathrm{H}, \mathrm{m}), 2.43-2.54(3 \mathrm{H}, \mathrm{m}), 2.78-2.87(1 \mathrm{H}, \mathrm{m}), 3.53-3.56(1 \mathrm{H}, \mathrm{m}), 3.78-3.83(1 \mathrm{H}, \mathrm{m}), 6.80$ 
(1H, br). ${ }^{13} \mathrm{C}$ NMR $\left(\mathrm{CDCl}_{3}\right) \delta 22.2,35.4,54.7,58.6,177.3$. EIMS $\mathrm{m} / z$ (rel intensity) $273\left(\mathrm{M}^{+}, 2\right), 245$ (2), 188 (4), 148 (33), 146 (100), 127 (7), 110 (28), 82 (28). Anal. calcd. for $\mathrm{C}_{6} \mathrm{H}_{9} \mathrm{ClINO}$ : C, 26.35; H, $3.32 ;$ N, 5.12. Found: C, 26.36; H, 3.48; N, 5.04.

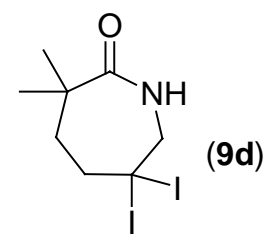

Method C: White solid. Yield: $72 \%$. Mp 135-137 ${ }^{\circ} \mathrm{C} .{ }^{1} \mathrm{H}$ NMR $\left(300 \mathrm{MHz}, \mathrm{CDCl}_{3}\right) \delta 1.16(6 \mathrm{H}, \mathrm{br}), 1.62$ (2H, br), $2.75(2 \mathrm{H}, \mathrm{br}), 3.58(2 \mathrm{H}, \mathrm{br}), 6.13(1 \mathrm{H}, \mathrm{br}) .{ }^{13} \mathrm{C} \mathrm{NMR}\left(\mathrm{CDCl}_{3}\right) \delta 14.1,22.7,29.4,29.6,30.2$, 31.9, 61.5, 179.8. EIMS: $\mathrm{m} / z$ (rel intensity) $394\left(\mathrm{M}^{+}+1,32\right), 266$ (30), 238 (13), 167 (6), 139 (28), 127( 13), 111 (100), 96 (69). HRMS calcd for $\mathrm{C}_{8} \mathrm{H}_{13} \mathrm{I}_{2} \mathrm{NO}\left(\mathrm{M}^{+}\right)$: 392.9087. Found: 392.9095.

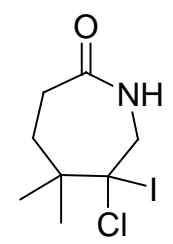

$(9 e)$

Method B: White solid. Mp 167-170 ${ }^{\circ} \mathrm{C}$. Yield: 77\%. ${ }^{1} \mathrm{H}$ NMR $\left(300 \mathrm{MHz}, \mathrm{CDCl}_{3}\right) \delta 1.37(6 \mathrm{H}, \mathrm{s}), 1.68-$ $1.87(2 \mathrm{H}, \mathrm{m}), 2.41-2.44(2 \mathrm{H}, \mathrm{m}), 3.68-3.80(2 \mathrm{H}, \mathrm{m}), 6.41(1 \mathrm{H}, \mathrm{br}) .{ }^{13} \mathrm{C} \mathrm{NMR}\left(\mathrm{CDCl}_{3}\right) \delta 22.3,31.1$, 31.9, 32.7, 33.1, 46.9, 55.1, 176.6. EIMS: $m / z$ (rel intensity) $301\left(\mathbf{M}^{+}, 3\right), 253$ (2), 210 (1), 174 (36), 145 (33), 127 (19), 98 (100), 67 (79). Anal. calcd for $\mathrm{C}_{8} \mathrm{H}_{13} \mathrm{ClINO}$ C, 31.86; H, 4.35; N, 4.64. Found: C, 31.98; H, 4.27; N, 4.42.

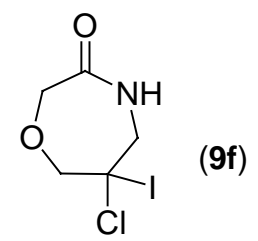

Method B: White solid. Mp 108-110 ${ }^{\circ} \mathrm{C}$. Yield: $52 \% .{ }^{1} \mathrm{H}$ NMR $\left(300 \mathrm{MHz}, \mathrm{CDCl}_{3}\right) \delta 3.74-3.81(1 \mathrm{H}, \mathrm{m})$, 3.92-3.99 $(1 \mathrm{H}, \mathrm{m}), 4.10-4.14(1 \mathrm{H}, \mathrm{m}), 4.27-4.33(3 \mathrm{H}, \mathrm{m}), 6.68(1 \mathrm{H}, \mathrm{br}) .{ }^{13} \mathrm{C}$ NMR $\left(\mathrm{CDCl}_{3}\right) \delta 52.4,57.1$, 73.6, 85.8, 173.6. EIMS: $m / z$ (rel intensity) $275\left(\mathrm{M}^{+}, 1\right), 256$ (1), 217 (1), 188 (13), 148 (10), 120 (25), 90 (100), 75 (20). HRMS calcd for $\mathrm{C}_{5} \mathrm{H}_{8} \mathrm{ClNO}_{2}\left(\mathrm{M}^{+}+1\right)$ : 275.9288. Found: 275.9278. Anal. calcd for $\mathrm{C}_{5} \mathrm{H}_{7} \mathrm{ClINO}_{2}$ : C, 21.80; H, 2.56; N, 5.08. Found: C, 21.97; H, 2.60; N, 4.93.<smiles>O=C(CCCC=CCCl)N=CCCCC(O)=NSc1ccccc1</smiles>

White solid. Mp 47-48 ${ }^{\circ} \mathrm{C}$. Yield: $61 \%$. Two isomers (2:1). ${ }^{1} \mathrm{H}$ NMR $\left(300 \mathrm{MHz}, \mathrm{CDCl}_{3}\right) \delta 1.69-1.83$ $(2 \mathrm{H}, \mathrm{m}), 2.05-2.08(1 \mathrm{H}, \mathrm{m}), 2.24-2.26(1 \mathrm{H}, \mathrm{m}), 2.33-2.40(1 \mathrm{H}, \mathrm{m}), 2.55-2.58(1 \mathrm{H}, \mathrm{m})$, 5.67-6.05 $(1 \mathrm{H}$, m), $6.64(1 \mathrm{H}, \mathrm{m}), 7.21-7.35(5 \mathrm{H}, \mathrm{m}) .{ }^{13} \mathrm{C} \mathrm{NMR}\left(\mathrm{CDCl}_{3}\right) \delta 24.1 / 24.6,26.3 / 30.1,35.4 / 35.7,117.9 / 119.1$, 123.4, 125.5/126.8, 129.0/129.3, 130.5/132.6, 138.7, 174.5/179.5. EIMS: $m / z$ (rel intensity) $255\left(\mathrm{M}^{+}\right.$, 2), 220 (2), 167 (2), 150 (4), 125 (100), 109 (23), 93 (24), 67 (31). Anal. calcd for $\mathrm{C}_{12} \mathrm{H}_{14} \mathrm{ClNOS}$ : C, 56.35; H, 5.52; N, 5.48. Found: C, 56.44; H, 5.52; N, 5.12. 


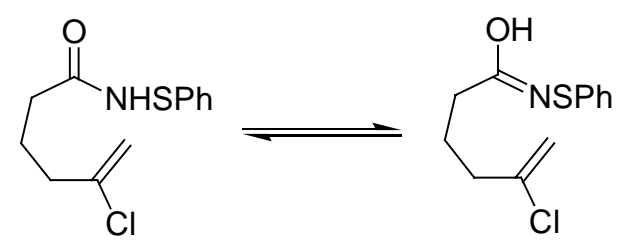

White solid. Mp 50-52 ${ }^{\circ} \mathrm{C}$. Yield: 52\%. Two isomers (2:1). ${ }^{1} \mathrm{H}$ NMR $\left(300 \mathrm{MHz}, \mathrm{CDCl}_{3}\right) \delta 1.88-2.01$ $(2 \mathrm{H}, \mathrm{m}), 2.39$ and $2.59(4 \mathrm{H}, \mathrm{m}), 5.09-5.18(2 \mathrm{H}, \mathrm{m}), 6.52$ and $6.93(1 \mathrm{H}, \mathrm{br}), 7.22-7.30(5 \mathrm{H}, \mathrm{m}) .{ }^{13} \mathrm{C}$ NMR $\left(\mathrm{CDCl}_{3}\right) \delta 22.0 / 22.8,29.5,34.8 / 38.1,112.7 / 113.1,123.4,125.9,126.8 / 127.1,129.1 / 129.3$, 138.6/141.6, 174.2. EIMS: $m / z$ (rel intensity) $255\left(\mathbf{M}^{+}, 1\right), 220$ (10), 159 (1), 125 (100), 109 (27), 93 (22), 67 (30), 55 (47). Anal. calcd for $\mathrm{C}_{12} \mathrm{H}_{14} \mathrm{ClNOS}$ : C, 56.35; H, 5.52; N, 5.48. Found: C, 56.34; $\mathrm{H}$, $5.43 ; \mathrm{N}, 5.12$.<smiles>[2H]C(Cl)C1CCCC(=O)N1</smiles>

White solid. Mp 127-129 ${ }^{\circ} \mathrm{C}$. Yield: $61 \% .{ }^{1} \mathrm{H}$ NMR (300MHz, $\left.\mathrm{CDCl}_{3}\right) \delta 1.43-1.56(1 \mathrm{H}, \mathrm{m}), 1.68-1.83$ $(1 \mathrm{H}, \mathrm{m}), 1.88-2.01(2 \mathrm{H}, \mathrm{m}), 2.26-2.47(2 \mathrm{H}, \mathrm{m}), 3.40$ and $3.57(1 \mathrm{H}, \mathrm{m}), 3.61-3.65(1 \mathrm{H}, \mathrm{m}), 6.12(1 \mathrm{H}$, br). ${ }^{13} \mathrm{C} \mathrm{NMR}\left(\mathrm{CDCl}_{3}\right) \delta 19.2,26.2,31.3,47.8(\mathrm{t}, J=18 \mathrm{~Hz}), 53.9,172.1$. EIMS: $\mathrm{m} / z$ (rel intensity) $148\left(\mathrm{M}^{+}, 2\right), 131$ (1), 98 (100), 79 (4), 70 (14), 55 (99), 44 (57). Anal. calcd for $\mathrm{C}_{6} \mathrm{H}_{9} \mathrm{DClNO}$ : C, 48.49 ; H, 6.78; N, 9.43. Found: C, 48.76; H, 6.86; N, 8.97.<smiles>NC(=O)CCCC=CCl</smiles>

(13)

White solid. $Z: E=50: 50 . \mathrm{Mp} \mathrm{54-56}{ }^{\circ} \mathrm{C}$. Yield: $18 \% .{ }^{1} \mathrm{H}$ NMR $\left(300 \mathrm{MHz}, \mathrm{CDCl}_{3}\right) \delta 1.66-1.84(2 \mathrm{H}, \mathrm{m})$, 2.09-2.33 (4H, m), $5.43(1 \mathrm{H}, \mathrm{br}), 5.72-6.08(2 \mathrm{H}, \mathrm{m}) .{ }^{13} \mathrm{C} \mathrm{NMR}\left(\mathrm{CDCl}_{3}\right) \delta 24.0 / 24.3,26.4 / 30.2$, 34.6/34.9, 117.8/119.0, 130.6/132.8, 174.6/174.9. EIMS: $\mathrm{m} / z$ (rel intensity) $149\left(\mathrm{M}^{+}+1,1\right), 129$ (1), 113 (45), 95 (8), 83 (6), 67 (26), 60 (100), 55 (39). Anal. calcd for $\mathrm{C}_{6} \mathrm{H}_{9} \mathrm{DClNO}$ : C, 48.49; H, 6.78; N, 9.43. Found: C, 48.56; H, 6.66; N, 9.42 .<smiles>[2H]C1(Cl)CCCC(=O)NC1</smiles>

White solid. Mp 90-92 ${ }^{\circ} \mathrm{C}$. Yield: 52\%. ${ }^{1} \mathrm{H}$ NMR $\left(300 \mathrm{MHz}, \mathrm{CDCl}_{3}\right) \delta 1.65-1.75(1 \mathrm{H}, \mathrm{m}), 1.91-2.06$ $(2 \mathrm{H}, \mathrm{m}), 2.25-2.33(1 \mathrm{H}, \mathrm{m}), 2.41-2.56(2 \mathrm{H}, \mathrm{m}), 3.38-3.57(2 \mathrm{H}, \mathrm{m}), 6.47(1 \mathrm{H}, \mathrm{br}) .{ }^{13} \mathrm{C} \mathrm{NMR}\left(\mathrm{CDCl}_{3}\right) \delta$ 20.6, 35.9, 39.6, 48.5, 57.4(t, $J=17 \mathrm{~Hz}), 178.1$. EIMS: $m / z$ (rel intensity) $148\left(\mathrm{M}^{+}, 1\right), 98(100), 83(2)$, 70 (14), 55 (95), 42 (38). Anal. calcd for $\mathrm{C}_{6} \mathrm{H}_{9} \mathrm{DCINO}$ : C, 48.49; H, 6.78; N, 9.43. Found: C, 48.83; $\mathrm{H}$, $6.88 ; \mathrm{N}, 9.12$.<smiles>C=C(Cl)CCCC(=O)N[O]</smiles> 
White solid. Mp 66-68 ${ }^{\circ} \mathrm{C}$. Yield: 31\%. ${ }^{1} \mathrm{H}$ NMR (300MHz, $\left.\mathrm{CDCl}_{3}\right) \delta 1.91-1.95(2 \mathrm{H}, \mathrm{m}), 2.25(2 \mathrm{H}, \mathrm{t}, J$ $=7.5 \mathrm{~Hz}), 2.42(2 \mathrm{H}, \mathrm{t}, J=7.5 \mathrm{~Hz}), 5.18(2 \mathrm{H}, \mathrm{d}, J=7.2 \mathrm{~Hz}), 5.49(1 \mathrm{H}, \mathrm{br}) .{ }^{13} \mathrm{C} \mathrm{NMR}\left(\mathrm{CDCl}_{3}\right) \delta 22.7$, 34.0, 38.2, 112.9, 141.8, 174.5. EIMS: $m / z$ (rel intensity) $149\left(\mathrm{M}^{+}+1,3\right), 113$ (44), 95 (5), 83 (5), 69 (24), 60 (100), 55 (33), 41 (68). Anal. calcd for $\mathrm{C}_{6} \mathrm{H}_{9}$ DCINO: C, 48.49; H, 6.78; N, 9.43. Found: C, 48.79; H, 6.91; N, 9.44.

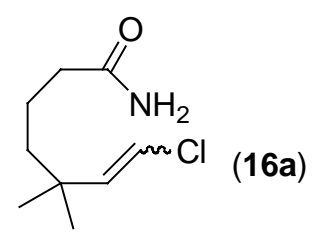

Yellowish oil. Yield: 56\%. $Z: E=23: 77 .{ }^{1} \mathrm{H}$ NMR (300MHz, $\left.\mathrm{CDCl}_{3}\right) \delta 1.01(1 \mathrm{H}, \mathrm{s}), 1.16(5 \mathrm{H}, \mathrm{s})$, 1.49-1.62 (4H, m), 2.14-2.21 $(2 \mathrm{H}, \mathrm{m}), 5.57-5.59(1 \mathrm{H}, \mathrm{m}), 5.62(1 \mathrm{H}, \mathrm{br}), 5.85-5.93(1 \mathrm{H}, \mathrm{m}), 5.97(1 \mathrm{H}$, br). ${ }^{13} \mathrm{C}$ NMR $\left(\mathrm{CDCl}_{3}\right) \quad \delta \quad 20.6 / 20.9,26.7 / 27.7,36.2 / 36.3,36.4 / 37.0,41.7 / 42.2,115.5 / 116.4$, 138.9/142.9, 175.6/175.8. EIMS: $m / z$ (rel intensity) $190\left(\mathrm{M}^{+}+1,15\right), 154$ (19), 137 (7), 109 (17), 95 (28), 86 (35), 67 (53), 59 (100). HRMS calcd for $\mathrm{C}_{9} \mathrm{H}_{16} \mathrm{ClNO}\left(\mathrm{M}^{+}\right)$: 189.0920. Found: 189.0912.

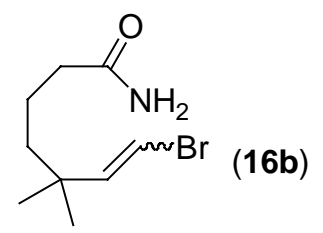

Yellowish oil. Yield: 53\%. $Z: E=33: 67 .{ }^{1} \mathrm{H}$ NMR $\left(300 \mathrm{MHz}, \mathrm{CDCl}_{3}\right) \delta 1.02(1 \mathrm{H}, \mathrm{s}), 1.19(5 \mathrm{H}, \mathrm{s})$, 1.53-1.66 (4H, m), 2.16-2.24 (2H, m), $5.55(1 \mathrm{H}, \mathrm{br}), 5.89(1 \mathrm{H}, \mathrm{br}), 5.94-6.16(2 \mathrm{H}, \mathrm{m}) .{ }^{13} \mathrm{C} \mathrm{NMR}$ $\left(\mathrm{CDCl}_{3}\right) \delta 20.6 / 20.9,26.5 / 27.6,36.2 / 36.4,37.0 / 38.5,41.5 / 42.0,102.9 / 104.5,141.8 / 147.0,175.3 / 175.5$. EIMS: $m / z$ (rel intensity) $234\left(\mathrm{M}^{+}+1,32\right), 154$ (81), 147 (13), 137 (33), 109 (33), 95 (77), 86 (64), 59 (100). Anal. calcd for $\mathrm{C}_{9} \mathrm{H}_{16} \mathrm{BrNO}$ : C, 46.17; H, 6.89; N, 5.98. Found: C, 46.21; H, 6.64; N, 5.79.

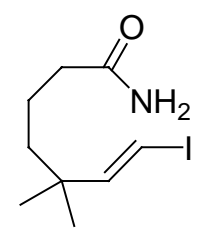

$(16 c)$

Yellowish oil. Yield: $58 \% .{ }^{1} \mathrm{H}$ NMR $\left(300 \mathrm{MHz}, \mathrm{CDCl}_{3}\right) \delta 1.25(6 \mathrm{H}, \mathrm{s}), 1.57-1.62(4 \mathrm{H}, \mathrm{m}), 2.17-2.24$ $(2 \mathrm{H}, \mathrm{m}), 5.54(1 \mathrm{H}, \mathrm{br}), 5.87(1 \mathrm{H}, \mathrm{br}), 6.13-6.16(1 \mathrm{H}, \mathrm{m}), 6.44-6.47(1 \mathrm{H}, \mathrm{m}) .{ }^{13} \mathrm{C} \mathrm{NMR}\left(\mathrm{CDCl}_{3}\right) \delta 20.8$, 27.6, 36.4, 37.5, 41.2, 75.3, 147.7, 175.6. EIMS: $m / z$ (rel intensity) $282\left(\mathrm{M}^{+}+1,4\right), 217$ (2), 195 (13), 154 (50), 137 (20), 109 (25), 95 (75), 59 (100). HRMS calcd for $\mathrm{C}_{9} \mathrm{H}_{16} \mathrm{INNaO}\left(\mathrm{M}^{+}+\mathrm{Na}\right): 304.0174$. Found: 304.0160.<smiles>CC1(C)CCCC(=O)NC1C(Cl)I</smiles>

(17a)

Method A: Colorless oil. Yield: $64 \%$. Two stereoisomers in $~ 80: 20$ ratio. ${ }^{1} \mathrm{H}$ NMR $\left(300 \mathrm{MHz}, \mathrm{CDCl}_{3}\right)$ $\delta$ 1.03-1.14 (6H, m), 1.46-1.56 (2H, m), 1.70-1.79 (2H, m), 2.42-2.54 (2H, m), 3.28/3.93 (1H, $2 \mathrm{dd}, J=$ 7.5, $1.5 \mathrm{~Hz}), 6.01(1 \mathrm{H}, \mathrm{br}), 6.07 / 6.36(1 \mathrm{H}, 2 \mathrm{~d}, J=2.1 \mathrm{~Hz}) .{ }^{13} \mathrm{C} \mathrm{NMR}\left(\mathrm{CDCl}_{3}\right) \delta 19.6,20.6,27.0,27.3$, 35.6, 38.1, 47.0, 69.6, 176.1. EIMS: $m / z$ (rel intensity) $316\left(\mathrm{M}^{+}+1,3\right), 254$ (1), 188 (52), 140 (6), 111 (20), 83 (49), 69 (96), 41 (100). HRMS calcd for $\mathrm{C}_{9} \mathrm{H}_{15} \mathrm{CIINO}\left(\mathrm{M}^{+}\right)$: 314.9887 . Found: 314.9896. 
<smiles>CC1(C)CCCC(=O)NC1C(Br)I</smiles>

(17b)

Method A: Yellowish oil. Yield: 70\%. Two stereoisomers in $~ 82: 18$ ratio. ${ }^{1} \mathrm{H}$ NMR $\left(300 \mathrm{MHz}, \mathrm{CDCl}_{3}\right)$ $\delta$ 1.04-1.14 $(6 \mathrm{H}, \mathrm{m}), 1.43-1.52(2 \mathrm{H}, \mathrm{m}), 1.70-1.80(2 \mathrm{H}, \mathrm{m}), 2.39-2.55(2 \mathrm{H}, \mathrm{m}), 3.48 / 3.96(1 \mathrm{H}, 2 \mathrm{dd}, J=$ $7.51 .5 \mathrm{~Hz}), 5.93(1 \mathrm{H}, \mathrm{br}), 5.96 / 6.12(1 \mathrm{H}, 2 \mathrm{~d}, J=1.8 \mathrm{~Hz}) .{ }^{13} \mathrm{C} \mathrm{NMR}\left(\mathrm{CDCl}_{3}\right) \delta 8.8,19.6 / 20.2,27.0$, 35.8, 38.5, 47.5, 69.3, 176.1. EIMS: $m / z$ (rel intensity) $360\left(\mathrm{M}^{+}+1,3\right), 280$ (3), 232 (22), 190 (2), 149 (12), 111 (29), 83 (43), 41 (100). HRMS calcd for $\mathrm{C}_{9} \mathrm{H}_{15} \mathrm{INO}\left(\mathrm{M}^{+}-\mathrm{Br}\right)$ ): 280.0198. Found: 280.0209.<smiles>CC1(C)CCCC(=O)NC1C(I)I</smiles>

(17c)

Method A: Yellowish oil. Yield: 60\%. ${ }^{1} \mathrm{H}$ NMR $\left(300 \mathrm{MHz}, \mathrm{CDCl}_{3}\right) \delta 1.00-1.03(3 \mathrm{H}, \mathrm{m}), 1.11-1.14(3 \mathrm{H}$, $\mathrm{m}), 1.21-1.41(2 \mathrm{H}, \mathrm{m}), 1.69-1.75(2 \mathrm{H}, \mathrm{m}), 2.41-2.54(2 \mathrm{H}, \mathrm{m}), 3.56-3.61(1 \mathrm{H}, \mathrm{m}), 5.62-5.66(1 \mathrm{H}, \mathrm{m})$, 5.81 (1H, br). ${ }^{13} \mathrm{C} \mathrm{NMR}\left(\mathrm{CDCl}_{3}\right) \delta-30.6,19.6,26.4,29.7,36.2,48.3,68.8,176.4$. EIMS: $\mathrm{m} / z$ (rel intensity) 407 ( $\left.\mathrm{M}^{+}, 20\right), 315$ (1), 279 (48), 237 (2), 169 (30), 127 (11), 111 (26), 69 (83). HRMS calcd for $\mathrm{C}_{9} \mathrm{H}_{15} \mathrm{I}_{2} \mathrm{NO}\left(\mathrm{M}^{+}\right)$: 406.9243 . Found: 406.9237.

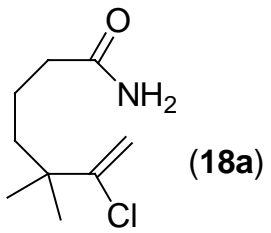

Yellowish solid. Yield: $61 \%$. Mp 52-54 ${ }^{\circ} \mathrm{C} .{ }^{1} \mathrm{H}$ NMR $\left(300 \mathrm{MHz}, \mathrm{CDCl}_{3}\right) \delta 1.17(6 \mathrm{H}, \mathrm{s}), 1.50-1.53(4 \mathrm{H}$, $\mathrm{m}), 2.15-2.32(2 \mathrm{H}, \mathrm{m}), 5.15-5.16(1 \mathrm{H}, \mathrm{m}), 5.20-5.21(1 \mathrm{H}, \mathrm{m}), 5.50(1 \mathrm{H}, \mathrm{br}), 5.72(1 \mathrm{H}, \mathrm{br}) .{ }^{13} \mathrm{C} \mathrm{NMR}$ $\left(\mathrm{CDCl}_{3}\right) \delta 20.6,26.7,36.2,39.7,41.7,111.5,151.0,175.3$. EIMS: $m / z$ (rel intensity) $190\left(\mathrm{M}^{+}+1,5\right)$, 154 (69), 128 (7), 111 (4), 95 (16), 86 (52), 67 (35), 59 (100). HRMS calcd for $\mathrm{C}_{9} \mathrm{H}_{16} \mathrm{NO}\left(\mathrm{M}^{+}-\mathrm{Cl}\right)$ : 154.1232. Found: 154.1230.

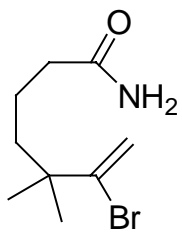

$(18 b)$

Yellowish solid. Yield: 63\%. Mp 62-64 ${ }^{\circ} \mathrm{C} .{ }^{1} \mathrm{H}$ NMR (300MHz, $\left.\mathrm{CDCl}_{3}\right) \delta 1.16(6 \mathrm{H}, \mathrm{s}), 1.44-1.55(4 \mathrm{H}$, m), 2.18-2.22 $(2 \mathrm{H}, \mathrm{m}), 5.46(1 \mathrm{H}, \mathrm{s}), 5.56(1 \mathrm{H}, \mathrm{s}), 5.58(1 \mathrm{H}, \mathrm{br}), 5.93(1 \mathrm{H}, \mathrm{br}) .{ }^{13} \mathrm{C} \mathrm{NMR}\left(\mathrm{CDCl}_{3}\right) \delta$ 20.5, 27.0, 36.1, 40.5, 42.5, 116.1, 145.1, 175.4. EIMS: $\mathrm{m} / z$ (rel intensity) $154\left(\mathrm{M}^{+}-\mathrm{Br}, 20\right), 137$ (6), 121 (2), 109 (8), 86 (27), 67 (32), 59 (100), 44 (57). HRMS calcd for $\mathrm{C}_{9} \mathrm{H}_{16} \mathrm{NO}\left(\mathrm{M}^{+}-\mathrm{Br}\right): 154.1232$. Found: 154.1231.

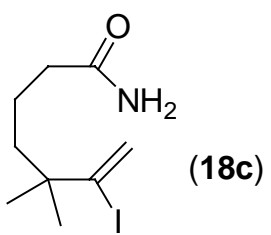

Yellowish oil. Yield: 53\%. ${ }^{1} \mathrm{H}$ NMR $\left(300 \mathrm{MHz}, \mathrm{CDCl}_{3}\right) \delta 1.17(6 \mathrm{H}, \mathrm{s}), 1.26-1.34(2 \mathrm{H}, \mathrm{m}), 1.39-1.52$ $(2 \mathrm{H}, \mathrm{m}), 2.20-2.25(2 \mathrm{H}, \mathrm{m}), 5.48(2 \mathrm{H}, \mathrm{br}), 5.83(1 \mathrm{H}, \mathrm{d}, J=2.4 \mathrm{~Hz}), 6.08(1 \mathrm{H}, \mathrm{d}, J=2.1 \mathrm{~Hz}) .{ }^{13} \mathrm{C} \mathrm{NMR}$ 
$\left(\mathrm{CDCl}_{3}\right) \delta 20.5,27.1,36.1,42.0,43.4,125.2,127.4,175.1$. EIMS: $m / z$ (rel intensity) $282\left(\mathrm{M}^{+}+1,1\right)$, 196 (1), 154 (63), 137 (15), 109 (24), 95 (48), 67 (70), 44 (100). HRMS calcd for $\mathrm{C}_{9} \mathrm{H}_{16} \mathrm{NO}\left(\mathrm{M}^{+}-\mathrm{I}\right)$ : 154.1232. Found: 154.1234.

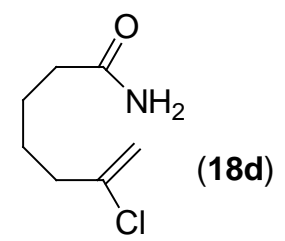

White solid. Yield: $75 \%$. Mp 97-99 ${ }^{\circ} \mathrm{C} .{ }^{1} \mathrm{H}$ NMR $\left(300 \mathrm{MHz}, \mathrm{CDCl}_{3}\right) \delta 1.61-1.69(4 \mathrm{H}, \mathrm{m}), 2.26(2 \mathrm{H}, \mathrm{t}, J$ $=6.9 \mathrm{~Hz}), 2.38(2 \mathrm{H}, J=6.9 \mathrm{~Hz}), 5.14-5.17(2 \mathrm{H}, \mathrm{m}), 5.48(1 \mathrm{H}, \mathrm{br}), 5.60(1 \mathrm{H}, \mathrm{br}) .{ }^{13} \mathrm{C} \mathrm{NMR}\left(\mathrm{CDCl}_{3}\right) \delta$ 24.2, 26.6, 35.5, 38.8, 112.3, 142.3, 175.3. EIMS: $m / z$ (rel intensity) $162\left(\mathrm{M}^{+}+1,2\right), 145(1), 126(17)$, 102 (1), 81 (12), 72 (16), 59 (100), 44 (58). Anal. calcd for $\mathrm{C}_{7} \mathrm{H}_{12} \mathrm{ClNO}$ : C, 52.02; H, 7.48; N, 8.67. Found: C, 51.97; H, 7.65; N, 8.65.

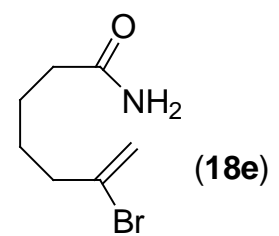

White solid. Yield: $77 \%$. Mp 90-92 ${ }^{\circ} \mathrm{C} .{ }^{1} \mathrm{H}$ NMR $\left(300 \mathrm{MHz}, \mathrm{CDCl}_{3}\right) \delta$ 1.57-1.68 (4H, m), 2.21-2.27 $(2 \mathrm{H}, \mathrm{m}), 2.45-2.47(2 \mathrm{H}, \mathrm{m}), 5.40(1 \mathrm{H}, \mathrm{s}), 5.59(1 \mathrm{H}, \mathrm{s}), 5.98(1 \mathrm{H}, \mathrm{br}), 6.48(1 \mathrm{H}, \mathrm{br}) .{ }^{13} \mathrm{C} \mathrm{NMR}\left(\mathrm{CDCl}_{3}\right)$ $\delta$ 24.0, 27.2 , 35.4, 40.9, 116.7, 133.9, 175.8. EIMS: $m / z$ (rel intensity) $206\left(\mathrm{M}^{+}+1,0.7\right), 126(100), 119$ (2), 109 (13), 93 (1), 84 (20), 67 (12), 59 (40). Anal. calcd for $\mathrm{C}_{7} \mathrm{H}_{12} \mathrm{BrNO}$ : C, 40.80; H, 5.87; N, 6.80. Found: C, 40.77; H, 5.81; N, 6.79.

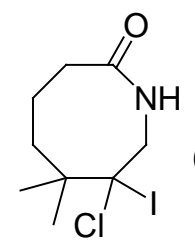

(19a)

Method C: White solid. Yield: 80\%. Mp 139-141 ${ }^{\circ} \mathrm{C} .{ }^{1} \mathrm{H}$ NMR $\left(300 \mathrm{MHz}, \mathrm{CDCl}_{3}\right) \delta 1.36(6 \mathrm{H}, \mathrm{br}), 1.64-$ $1.71(2 \mathrm{H}, \mathrm{m}), 2.42(2 \mathrm{H}, \mathrm{br}), 4.09(2 \mathrm{H}, \mathrm{br}), 6.41(1 \mathrm{H}, \mathrm{br}) .{ }^{13} \mathrm{C} \mathrm{NMR}\left(\mathrm{CDCl}_{3}\right) \delta 23.3,29.7,31.9,32.2$, 38.9, 46.7, 58.9, 175.2. EIMS: $m / z$ (rel intensity) $315\left(\mathbf{M}^{+}, 2\right), 253$ (1), 188 (18), 159 (52), 123 (50), 95 (100), 67 (59), 55 (97). HRMS calcd for $\mathrm{C}_{9} \mathrm{H}_{15} \mathrm{ClNO}\left(\mathrm{M}^{+}-\mathrm{I}\right)$ : 188.0842 . Found: 188.0847.

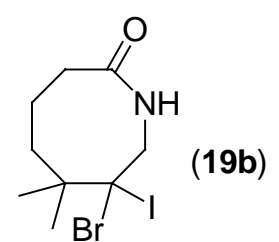

Method C: White solid. Yield: $72 \%$. Mp 156-157 ${ }^{\circ} \mathrm{C} .{ }^{1} \mathrm{H}$ NMR $\left(300 \mathrm{MHz}, \mathrm{CDCl}_{3}\right) \delta 1.38(6 \mathrm{H}, \mathrm{br}), 1.91$ (2H. br), 2.44 (2H, br), 4.16 (2H, br), 6.40 (1H, br). ${ }^{13} \mathrm{C}$ NMR $\left(\mathrm{CDCl}_{3}\right) \delta 23.4,29.7,31.6,31.9,38.9$, 46.3, 62.1, 175.2. EIMS: $m / z$ (rel intensity) $359\left(\mathrm{M}^{+}, 2\right), 253$ (1), 232 (8), 203 (21), 152 (33), 123 (60), 95 (100), 67 (69). HRMS calcd for $\mathrm{C}_{9} \mathrm{H}_{15} \mathrm{BrNO}\left(\mathrm{M}^{+}-\mathrm{I}\right)$ : 232.0337. Found: 232.0340. 


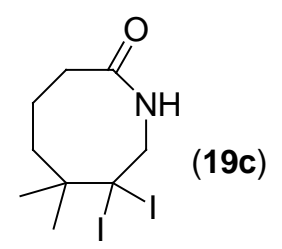

Method C: Yellowish solid. Yield: $45 \%$. Mp 150-152 ${ }^{\circ} \mathrm{C} .{ }^{1} \mathrm{H}$ NMR $\left(300 \mathrm{MHz}, \mathrm{CDCl}_{3}\right) \delta 1.27(6 \mathrm{H}, \mathrm{br})$, 1.99 (2H, br), 2.44 (2H, br), $4.15(2 \mathrm{H}, \mathrm{br}), 6.41$ (1H, br). ${ }^{13} \mathrm{C} \mathrm{NMR}\left(\mathrm{CDCl}_{3}\right) \delta 20.2,29.7,31.3,31.7$, 38.6, 46.1, 60.2, 175.5. EIMS: $m / z$ (rel intensity) $407\left(\mathrm{M}^{+}, 2\right), 377$ (12), 280 (20), 251 (29), 152 (26), 124 (86), 105 (48), 41 (100). HRMS calcd for $\mathrm{C}_{9} \mathrm{H}_{15} \mathrm{I}_{2} \mathrm{NO}\left(\mathrm{M}^{+}\right)$: 406.9243 . Found: 406.6232.

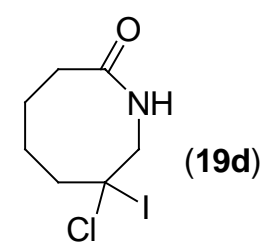

Method A: White solid. Yield: $80 \%$. Mp $113-115^{\circ} \mathrm{C} .{ }^{1} \mathrm{H}$ NMR $\left(300 \mathrm{MHz}, \mathrm{CDCl}_{3}\right) \delta 1.21-1.48(1 \mathrm{H}, \mathrm{m})$, 1.60-2.14 (4H, m), 2.32-2.48 (2H, m), 2.64-2.75 (1H, m), 3.59-4.22 (2H, m), $6.67(1 \mathrm{H}, \mathrm{br}){ }^{13} \mathrm{C}$ NMR $\left(\mathrm{CDCl}_{3}\right) \delta 21.0,25.0,26.2 / 26.8,33.5,47.9 / 49.7,58.4 / 60.5,176.9$. EIMS: $m / z$ (rel intensity) $288\left(\mathrm{M}^{+}+1\right.$, 13), 252 (1), 160 (100), 124 (15), 96 (33), 82 (12), 67 (36), 55(35). Anal. calcd for $\mathrm{C}_{7} \mathrm{H}_{11} \mathrm{ClINO}$ : C, 29.24; H, 3.86; N, 4.87. Found: C, 29.31; H, 3.95; N, 4.65.

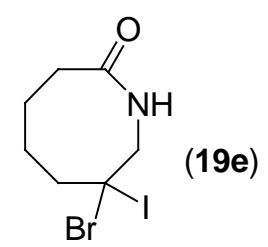

Method A: White solid. Yield: $85 \%$. Mp 136-138 ${ }^{\circ} \mathrm{C} .{ }^{1} \mathrm{H}$ NMR $\left(300 \mathrm{MHz}, \mathrm{CDCl}_{3}\right) \delta 1.29-1.45(1 \mathrm{H}, \mathrm{m})$, 1.69-1.95 (4H, m), 2.33-2.36 (2H, m), 2.74-2.95 (1H, m), 3.80-4.26 (2H, m), $6.33(1 \mathrm{H}, \mathrm{br}) .{ }^{13} \mathrm{C} \mathrm{NMR}$ $\left(\mathrm{CDCl}_{3}\right) \delta 26.1 / 26.5,26.7 / 27.2,33.7,46.3 / 47.3 .49 .1 / 50.3,58.8 / 59.1,176.6$. EIMS: $\mathrm{m} / z$ (rel intensity) $204\left(\mathrm{M}^{+}-127,74\right), 176$ (4), 160 (5), 147 (4), 125 (23), 96 (42), 79 (54), 67 (73). Anal. calcd for $\mathrm{C}_{7} \mathrm{H}_{11}$ BrINO: C, 25.33; H, 3.34; N, 4.22. Found: $\mathrm{C}, 25.50 ; \mathrm{H}, 3.23 ; \mathrm{N}, 3.81$. The structure was further confirmed by its X-ray diffraction analysis.

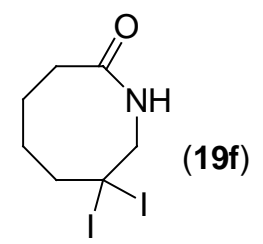

Method A: White solid. Yield: 79\%. Mp 175-178 ${ }^{\circ} \mathrm{C} .{ }^{1} \mathrm{H}$ NMR $\left(300 \mathrm{MHz}, \mathrm{CDCl}_{3}\right) \delta 1.15-1.26(1 \mathrm{H}, \mathrm{m})$, 1.65-1.83 (2H, m), 1.90-1.95 (1H, m), 2.40-2.49 (2H, m), 2.53-2.64 (1H, m), 2.87-2.96 $(1 \mathrm{H}, \mathrm{m}) .{ }^{13} \mathrm{C}$ NMR $\left(\mathrm{CDCl}_{3}\right) \delta 1.0,26.2,28.3,34.1,51.7,60.5,176.3$. EIMS: $m / z$ (rel intensity) $379\left(\mathrm{M}^{+}, 0.7\right), 252$ (100), 223 (2), 167 (3), 125 (37), 96 (23), 81 (40), 69 (36). HRMS calcd for $\mathrm{C}_{7} \mathrm{H}_{12} \mathrm{I}_{2} \mathrm{NO}\left(\mathrm{M}^{+}+1\right)$ : 379.9008. Found: 379.9004 . The structure was further confirmed by its X-ray diffraction analysis.

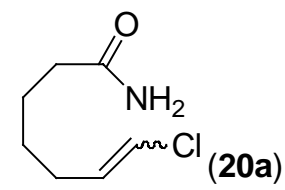

White solid. Yield: $75 \%$. Mp 64-66 ${ }^{\circ} \mathrm{C} . Z: E=25: 75 .{ }^{1} \mathrm{H}$ NMR $\left(300 \mathrm{MHz}, \mathrm{CDCl}_{3}\right) \delta 1.43-1.54(2 \mathrm{H}, \mathrm{m})$, 1.64-1.74 (2H, m), 2.05-2.29 (4H, m), $5.48(1 \mathrm{H}, \mathrm{br}), 5.53(1 \mathrm{H}, \mathrm{br}), 5.73-6.06(2 \mathrm{H}, \mathrm{m}) .{ }^{13} \mathrm{C} \mathrm{NMR}$ 
$\left(\mathrm{CDCl}_{3}\right) \delta 24.8,26.6,27.8,35.5,118.5,131.2,175.2$. EIMS: $\mathrm{m} / z$ (rel intensity) $162\left(\mathrm{M}^{+}+1,14\right), 126$ (32), 109 (4), 99 (6), 81 (14), 72 (60), 59 (83), 44 (100). Anal. calcd for $\mathrm{C}_{7} \mathrm{H}_{12} \mathrm{ClNO}$ : C, 52.02; H, 7.48; N, 8.67. Found: C, 51.92; H, 7.68; N, 8.53.

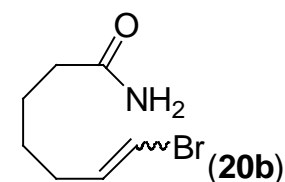

White solid. Yield: $80 \%$. Mp 63-65 ${ }^{\circ} \mathrm{C} . Z: E=20: 80 .{ }^{1} \mathrm{H}$ NMR $\left(300 \mathrm{MHz}, \mathrm{CDCl}_{3}\right) \delta 1.43-1.49(2 \mathrm{H}, \mathrm{m})$, 1.64-1.74 (2H, m), 2.20-2.28 (4H, m), $5.46(1 \mathrm{H}, \mathrm{br}), 5.65(1 \mathrm{H}, \mathrm{br}), 6.06-6.19(2 \mathrm{H}, \mathrm{m}) .{ }^{13} \mathrm{C}$ NMR $\left(\mathrm{CDCl}_{3}\right) \delta 24.7 / 24.8,27.5 / 28.1,29.3 / 32.6,35.5,108.2$, 134.3, 175.3. EIMS: $m / z$ (rel intensity) 206 $\left(\mathrm{M}^{+}+1,2\right), 126$ (37), 109 (5), 93 (2), 86 (5), 72 (48), 59 (98), 44 (100). Anal. calcd for $\mathrm{C}_{7} \mathrm{H}_{12} \mathrm{BrNO}: \mathrm{C}$, 40.80; H, 5.87; N, 6.80. Found: C, 40.77; H, 5.79; N, 6.81.

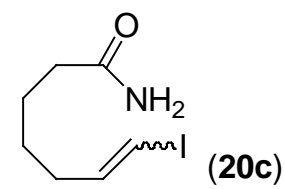

White solid. Yield: $70 \%$. Mp 63-65 ${ }^{\circ} \mathrm{C} . Z: E=10: 90 .{ }^{1} \mathrm{H}$ NMR $\left(300 \mathrm{MHz}, \mathrm{CDCl}_{3}\right) \delta 1.45-1.53(2 \mathrm{H}, \mathrm{m})$, 1.63-1.72 (2H, m), 2.14-2.28 (4H, m), $5.45(2 \mathrm{H}, \mathrm{br}), 6.13-6.23(2 \mathrm{H}, \mathrm{br}) .{ }^{13} \mathrm{C}$ NMR $\left(\mathrm{CDCl}_{3}\right) \delta 24.8,27.4$, 34.3, 35.6, 82.8, 140.7, 175.2. EIMS: $m / z$ (rel intensity) $254\left(\mathrm{M}^{+}+1,5\right), 167$ (6), 126 (58), 109 (13), 81 (23), 59 (53), 44 (100). Anal. calcd for $\mathrm{C}_{7} \mathrm{H}_{12} \mathrm{INO}$ : C, 33.22; H, 4.78; N, 5.53. Found: C, 33.67; H, $4.94 ; \mathrm{N}, 5.40$.<smiles>O=C1CCCC(C=CCl)O1</smiles>

Method B: Colorless oil. Yield: $82 \% . Z: E=1: 1 .{ }^{1} \mathrm{H}$ NMR $\left(300 \mathrm{MHz}, \mathrm{CDCl}_{3}\right) \delta 1.65-1.78(1 \mathrm{H}, \mathrm{m})$, 1.88-2.13 (3H, m), 2.45-2.72 (2H, m), 4.83-4.94 (0.5H, m), 5.30-5.37 $(0.5 \mathrm{H}, \mathrm{m}), 5.92-6.11(1 \mathrm{H}, \mathrm{m})$, 6.21-6.47 (1H, m). ${ }^{13} \mathrm{C}$ NMR $\left(\mathrm{CDCl}_{3}\right) \delta 18.2 / 18.4,27.0 / 28.2,29.4 / 29.5,76.1 / 78.3,120.7 / 122.3$, 130.2/131.2, 170.5/171.1. ESI-MS: $m / z$ (rel intensity) $161\left(\mathrm{M}^{+}+1\right), 183\left(\mathrm{M}^{+}+\mathrm{Na}\right)$. HRMS calcd for $\mathrm{C}_{7} \mathrm{H}_{9} \mathrm{O}_{2}\left(\mathrm{M}^{+}-\mathrm{Cl}\right):$ 125.0603. Found: 125.0607 .<smiles>O=C1CCCC(C=CBr)O1</smiles>

Method B: Colorless oil. Yield: 69\%. $Z: E=1: 1 .{ }^{1} \mathrm{H}$ NMR $\left(300 \mathrm{MHz}, \mathrm{CDCl}_{3}\right) \delta 1.84-2.04$ (4H, m), 2.41-2.58 (2H, m), 4.72-7.79 $(0.5 \mathrm{H}, \mathrm{m}), 5.12-5.20(0.5 \mathrm{H}, \mathrm{m}), 6.15-6.23(1 \mathrm{H}, \mathrm{m}), 6.29(0.5 \mathrm{H}, \mathrm{dd}, J=$ 7.5, $0.6 \mathrm{~Hz}), 6.43(0.5 \mathrm{H}, \mathrm{dd}, J=13.5,0.6 \mathrm{~Hz}) .{ }^{13} \mathrm{C} \mathrm{NMR}\left(\mathrm{CDCl}_{3}\right) \delta$ 18.0/18.3, 26.7/27.8, 29.3/29.4, 78.4/79.4, 109.7/109.9, 133.1/135.1, 170.3/170.7. ESI-MS: $m / z$ (rel intensity) $205\left(\mathrm{M}^{+}+1\right), 227$ $\left(\mathrm{M}^{+}+\mathrm{Na}\right)$. Anal. calcd for $\mathrm{C}_{7} \mathrm{H}_{9} \mathrm{BrO}_{2}$ : $\mathrm{C}, 41.00 ; \mathrm{H}, 4.42$. Found: $\mathrm{C}, 39.61 ; \mathrm{H}, 4.73$.<smiles>O=C1CCCC(C=CI)O1</smiles>

Method B: Yellowish oil. Yield: 37\%. $Z: E=1: 1.8 .{ }^{1} \mathrm{H}$ NMR $\left(300 \mathrm{MHz}, \mathrm{CDCl}_{3}\right) \delta 1.61-1.74(1 \mathrm{H}, \mathrm{m})$, 1.85-2.11 (3H, m), 2.44-2.71 (2H, m), $4.78(0.36 \mathrm{H}, \mathrm{dt}, J=9.9,3.6 \mathrm{~Hz}), 5.00-5.07(0.64 \mathrm{H}, \mathrm{m}), 6.37-$ $6.58(2 \mathrm{H}, \mathrm{m}) .{ }^{13} \mathrm{C} \mathrm{NMR}\left(\mathrm{CDCl}_{3}\right) \delta 18.0 / 18.4,26.6 / 27.6,29.4 / 29.7,79.9 / 81.1,82.7 / 83.7,139.1 / 143.1$, 
170.2/170.6. EIMS: $m / z$ (rel intensity) $252\left(\mathrm{M}^{+}, 2\right), 235$ (1), 180 (13), 167 (1), 125 (33), 97 (100), 70 (20), 55 (77). HRMS calcd for $\mathrm{C}_{7} \mathrm{H}_{9} \mathrm{IO}_{2}\left(\mathrm{M}^{+}\right)$: 251.9647 . Found: 251.9658 .

\section{References for known compounds}

\begin{tabular}{|c|l|}
\hline Compound & Reference \\
\hline $\mathbf{2}$ & Shechter, H.; Kirk J. C. J. Am. Chem. Soc. 1951, 73, 3087. \\
\hline $\mathbf{3}$ & Matallana, A.; Kruger, A. W.; Kingsbury, C. A. J. Org. Chem. 1994, 59, 3020. \\
\hline $\mathbf{4}$ & $\begin{array}{l}\text { Hodjat-Kachani, H.; Lattes, A.; Perie, J. J.; Roussel, J. } \\
\text { J. Organomet. Chem. 1975, 96, 175. }\end{array}$ \\
\hline $\mathbf{5}$ & Curini, M.; Epifano, F.; Marcotullio, M. C.; Montanari, F. Synlett. 2004, 368. \\
\hline $\mathbf{6 a , ~ 8 a , ~ 1 8 f}$ & Hu, T.; Li, C. Org. Lett. 2005, 7, 2035. \\
\hline
\end{tabular}

All calculations were performed with the Gaussian 98 program $^{1}$ using the density functional theory method of B3LYP. ${ }^{2}$ The geometries were fully optimized at $6-31 \mathrm{G}^{*}$ level.

\section{References:}

1. Gaussian 98, Revision A.7, M. J. Frisch, G. W. Trucks, H. B. Schlegel, G. E. Scuseria, M. A. Robb, J. R. Cheeseman, V. G. Zakrzewski, J. A. Montgomery, Jr., R. E. Stratmann, J. C. Burant, S. Dapprich, J. M. Millam, A. D. Daniels, K. N. Kudin, M. C. Strain, O. Farkas, J. Tomasi, V. Barone, M. Cossi, R. Cammi, B. Mennucci, C. Pomelli, C. Adamo, S. Clifford, J. Ochterski, G. A. Petersson, P. Y. Ayala, Q. Cui, K. Morokuma, D. K. Malick, A. D. Rabuck, K. Raghavachari, J. B. Foresman, J. Cioslowski, J. V. Ortiz, A. G. Baboul, B. B. Stefanov, G. Liu, A. Liashenko, P. Piskorz, I. Komaromi, R. Gomperts, R. L. Martin, D. J. Fox, T. Keith, M. A. Al-Laham, C. Y. Peng, A. Nanayakkara, C. Gonzalez, M. Challacombe, P. M. W. Gill, B. Johnson, W. Chen, M. W. Wong, J. L. Andres, C. Gonzalez, M. Head-Gordon, E. S. Replogle, and J. A. Pople, Gaussian, Inc.: Pittsburgh PA, 1998.

2. (a) Becke, A. D. Phys. Rev. A 1988, 38, 3098. (b) Becke, A. D. J. Chem. Phys. 1993, 98, 1372, 5648. (v) Lee, C.; Yang, W.; Parr, R. G. Phys. Rev. B 1988, 37, 785.

$22(\mathrm{R}=\mathrm{Cl})$-radical

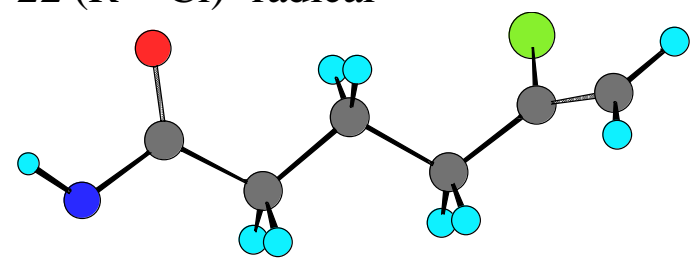

$\%$ chk=22 $(\mathbf{R}=\mathbf{C l})$-radical

\# ub3lyp/6-31g* opt freq=noraman

calculate for $22(\mathrm{R}=\mathrm{Cl})$-radical $\mathrm{HF}=-824.149327$

02

$\mathrm{N}$ 


$\begin{array}{lllllll}\mathrm{C} & 1 & 1.375627 & & & & \\ \mathrm{C} & 2 & 1.512661 & 1 & 116.967731 & & \\ \mathrm{O} & 2 & 1.237469 & 1 & 118.700777 & 3 & 173.025412 \\ \mathrm{C} & 3 & 1.530193 & 2 & 113.561032 & 1 & 175.660368 \\ \mathrm{C} & 5 & 1.539643 & 3 & 111.678654 & 2 & 178.539329 \\ \mathrm{C} & 6 & 1.504320 & 5 & 113.967701 & 3 & 179.500590 \\ \mathrm{C} & 7 & 1.331017 & 6 & 126.289079 & 5 & 112.294809 \\ \mathrm{Cl} & 7 & 1.774089 & 6 & 114.377153 & 5 & -67.645359 \\ \mathrm{H} & 3 & 1.098321 & 2 & 107.581195 & 1 & 51.908520 \\ \mathrm{H} & 1 & 1.028622 & 2 & 108.313064 & 4 & -30.124611 \\ \mathrm{H} & 3 & 1.100465 & 2 & 107.385247 & 1 & -60.896329 \\ \mathrm{H} & 5 & 1.096298 & 3 & 109.867967 & 2 & 56.836727 \\ \mathrm{H} & 5 & 1.094518 & 3 & 109.375583 & 2 & -59.443071 \\ \mathrm{H} & 6 & 1.097688 & 5 & 109.729500 & 3 & -59.860375 \\ \mathrm{H} & 6 & 1.098664 & 5 & 109.443025 & 3 & 57.118040 \\ \mathrm{H} & 8 & 1.084043 & 7 & 122.542056 & 6 & -179.750152 \\ \mathrm{H} & 8 & 1.086385 & 7 & 119.678973 & 6 & 0.340457\end{array}$

\section{$22(\mathrm{R}=\mathrm{Cl}) \mathrm{TS}(6-\mathrm{exo})$}

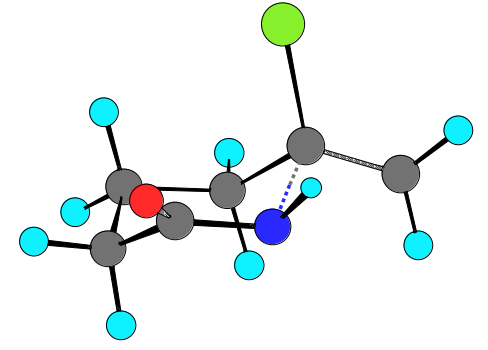

\%chk=22-Cl-6-exo

\# ub3lyp/6-31g* opt=(ts,noeigen,readfc,nofreeze) freq=noraman

calculate for 22-Cl-6-exo $\quad \mathrm{HF}=-824.137489$

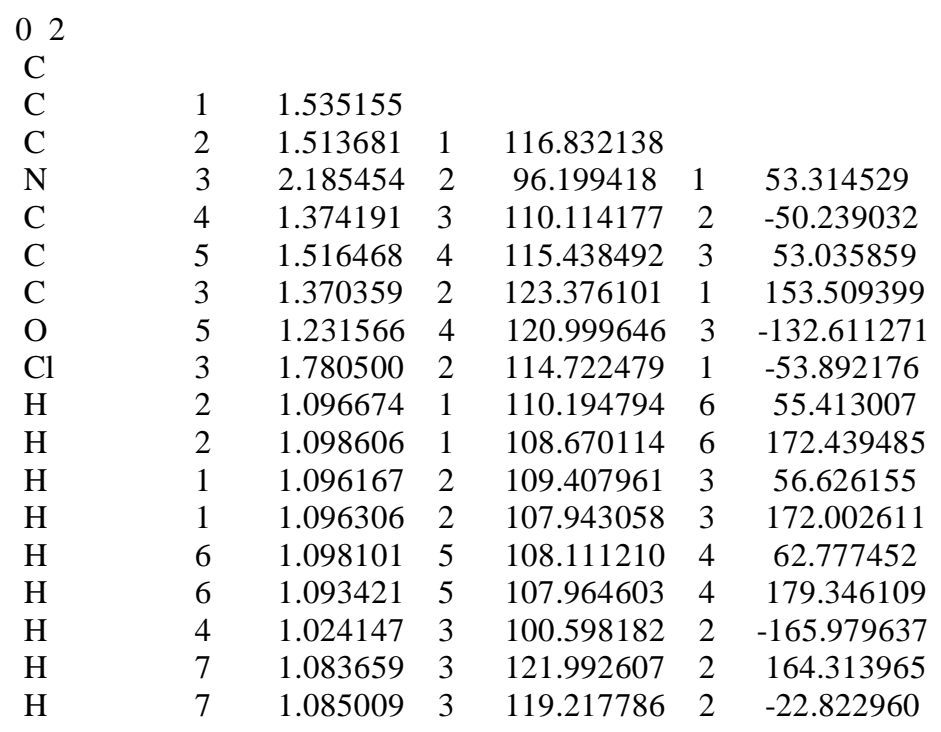

$22(\mathrm{R}=\mathrm{Cl}) \mathrm{TS}$ (7-endo) 


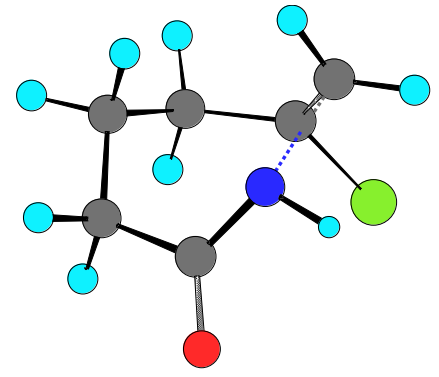

$\%$ chk=22-Cl-7-endo

\# ub3lyp/6-31g* opt=(ts,noeigen,readfc,nofreeze) freq=noraman

calculate for 22-Cl-7-endo $\quad \mathrm{HF}=-824.142949$

$\begin{array}{lllllll}02 & & & & & & \\ \mathrm{C} & & & & & & \\ \mathrm{C} & 1 & 1.508164 & & & & \\ \mathrm{~N} & 1 & 2.846320 & 2 & 100.216065 & & \\ \mathrm{C} & 2 & 1.542444 & 1 & 112.966556 & 3 & 11.814137 \\ \mathrm{C} & 3 & 1.375107 & 1 & 87.576629 & 2 & 48.577430 \\ \mathrm{C} & 5 & 1.528649 & 3 & 118.824339 & 1 & -59.235453 \\ \mathrm{O} & 5 & 1.233835 & 3 & 120.857384 & 1 & 128.944106 \\ \mathrm{C} & 1 & 1.355735 & 2 & 124.144952 & 4 & -38.703439 \\ \mathrm{Cl} & 1 & 1.754866 & 2 & 116.461523 & 4 & 136.379479 \\ \mathrm{H} & 2 & 1.094813 & 1 & 109.338496 & 8 & -161.051485 \\ \mathrm{H} & 4 & 1.095400 & 2 & 110.402618 & 1 & 58.198074 \\ \mathrm{H} & 2 & 1.100065 & 1 & 108.937812 & 8 & 83.248636 \\ \mathrm{H} & 4 & 1.097040 & 2 & 107.564369 & 1 & 173.587929 \\ \mathrm{H} & 6 & 1.098795 & 5 & 105.727747 & 3 & -98.221410 \\ \mathrm{H} & 6 & 1.096207 & 5 & 106.351211 & 3 & 149.771679 \\ \mathrm{H} & 3 & 1.026040 & 1 & 107.822381 & 2 & 155.806459 \\ \mathrm{H} & 8 & 1.083939 & 1 & 121.441635 & 2 & -179.873713 \\ \mathrm{H} & 8 & 1.081806 & 1 & 119.920893 & 2 & -14.702466\end{array}$

$22(\mathrm{R}=\mathrm{Cl}) \mathrm{TS}(1,5-\mathrm{H}$ migration $)$

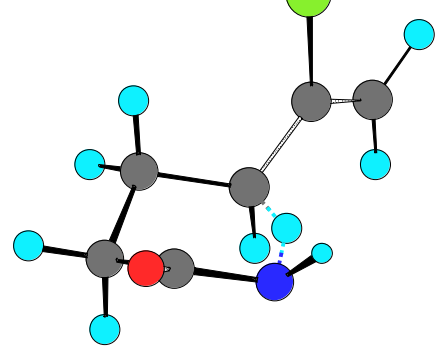

$\%$ chk=22-Cl-1,5-H

\# ub3lyp/6-31g* opt=(ts,noeigen,readfc,nofreeze) freq=noraman

calculate for $22-\mathrm{Cl}-1,5-\mathrm{H} \quad \mathrm{HF}=-824.134882$

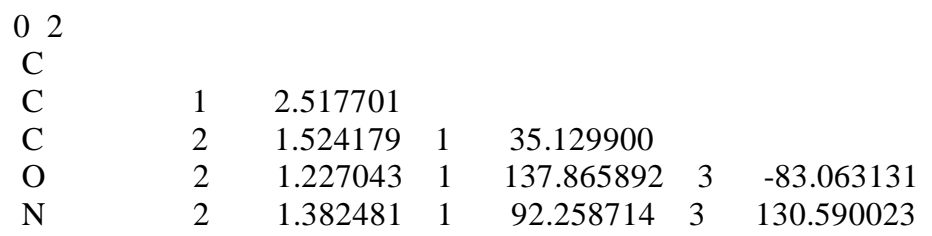




$\begin{array}{llllcll}\mathrm{C} & 1 & 1.531654 & 2 & 90.206348 & 4 & 148.838914 \\ \mathrm{C} & 6 & 1.472473 & 1 & 120.406333 & 2 & -127.524086 \\ \mathrm{C} & 7 & 1.341345 & 6 & 125.133776 & 1 & -136.765679 \\ \mathrm{Cl} & 7 & 1.771185 & 6 & 116.187976 & 1 & 46.103987 \\ \mathrm{H} & 3 & 1.092372 & 2 & 108.182378 & 1 & -121.912682 \\ \mathrm{H} & 3 & 1.098049 & 2 & 108.880708 & 1 & 120.766571 \\ \mathrm{H} & 1 & 1.097585 & 2 & 144.909700 & 4 & -84.386262 \\ \mathrm{H} & 6 & 1.239759 & 1 & 101.423607 & 2 & -6.436119 \\ \mathrm{H} & 6 & 1.095975 & 1 & 112.175681 & 2 & 100.767527 \\ \mathrm{H} & 1 & 1.094948 & 2 & 91.834852 & 4 & 39.063918 \\ \mathrm{H} & 8 & 1.083788 & 7 & 122.351916 & 6 & -178.017568 \\ \mathrm{H} & 8 & 1.085949 & 7 & 119.706045 & 6 & 2.305999 \\ \mathrm{H} & 5 & 1.024763 & 2 & 111.263934 & 1 & 122.478011\end{array}$

\section{$23(\mathrm{R}=\mathrm{Cl})$-radical}

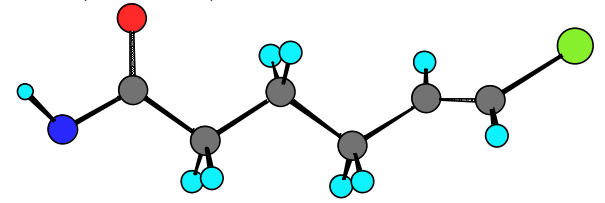

\%chk $=23(\mathbf{R}=\mathbf{C l})$-radical

\# ub3lyp/6-31g* opt freq=noraman

calculate for $23(\mathrm{R}=\mathrm{Cl})$-radical $\mathrm{HF}=-824.148182$

\begin{tabular}{lllllll}
02 & \multicolumn{7}{l}{} \\
$\mathrm{N}$ & & & & & & \\
$\mathrm{C}$ & 1 & 1.375516 & & & & \\
$\mathrm{C}$ & 2 & 1.512637 & 1 & 116.989071 & & \\
$\mathrm{O}$ & 2 & 1.237507 & 1 & 118.691827 & 3 & 172.857748 \\
$\mathrm{C}$ & 3 & 1.530216 & 2 & 113.599970 & 1 & 177.711123 \\
$\mathrm{C}$ & 5 & 1.540001 & 3 & 112.196193 & 2 & 179.789391 \\
$\mathrm{C}$ & 6 & 1.505074 & 5 & 112.546281 & 3 & 178.058768 \\
$\mathrm{C}$ & 7 & 1.330387 & 6 & 123.296650 & 5 & 119.786314 \\
$\mathrm{Cl}$ & 8 & 1.753017 & 7 & 123.388319 & 6 & -179.780530 \\
$\mathrm{H}$ & 3 & 1.098619 & 2 & 107.528858 & 1 & 54.017037 \\
$\mathrm{H}$ & 1 & 1.028578 & 2 & 108.383552 & 4 & -30.447383 \\
$\mathrm{H}$ & 3 & 1.100088 & 2 & 107.560116 & 1 & -58.805919 \\
$\mathrm{H}$ & 5 & 1.096467 & 3 & 109.793429 & 2 & 57.715034 \\
$\mathrm{H}$ & 5 & 1.096368 & 3 & 109.190048 & 2 & -58.052235 \\
$\mathrm{H}$ & 6 & 1.097999 & 5 & 109.904507 & 3 & -59.117442 \\
$\mathrm{H}$ & 6 & 1.100791 & 5 & 108.893907 & 3 & 57.045817 \\
$\mathrm{H}$ & 8 & 1.084791 & 7 & 124.078602 & 6 & 0.498589 \\
$\mathrm{H}$ & 7 & 1.088965 & 6 & 117.269673 & 5 & -59.611283
\end{tabular}

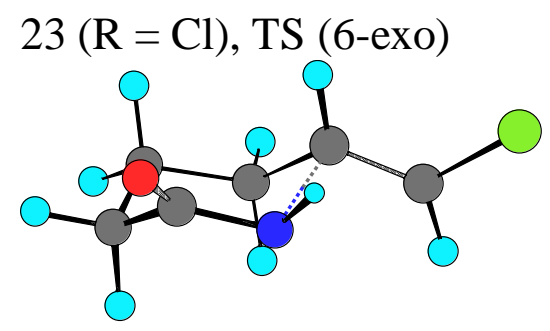

$\%$ chk=23-Cl-6-exo

\# ub3lyp/6-31g* opt=(ts, noeigen,readfc,nofreeze) freq=noraman 
calculate for 23-Cl-6-exo $\quad \mathrm{HF}=-824.143349$

\begin{tabular}{llllccc}
02 & \multicolumn{7}{c}{${ }^{2}$} & & & & & \\
$\mathrm{C}$ & & & & & \\
$\mathrm{C}$ & 1 & 1.538072 & & & & \\
$\mathrm{C}$ & 2 & 1.509735 & 1 & 114.442581 & & \\
$\mathrm{~N}$ & 3 & 2.303896 & 2 & 97.002902 & 1 & 53.678922 \\
$\mathrm{C}$ & 4 & 1.372107 & 3 & 105.815523 & 2 & -51.803755 \\
$\mathrm{C}$ & 5 & 1.517596 & 4 & 115.441437 & 3 & 54.894869 \\
$\mathrm{C}$ & 3 & 1.355956 & 2 & 122.324537 & 1 & 156.238979 \\
$\mathrm{O}$ & 5 & 1.234175 & 4 & 121.313249 & 3 & -130.222944 \\
$\mathrm{Cl}$ & 7 & 1.737586 & 3 & 122.718944 & 2 & 171.093238 \\
$\mathrm{H}$ & 2 & 1.096915 & 1 & 110.352262 & 6 & 55.971539 \\
$\mathrm{H}$ & 2 & 1.100206 & 1 & 107.901792 & 6 & 172.041744 \\
$\mathrm{H}$ & 1 & 1.098980 & 2 & 109.162175 & 3 & 54.833075 \\
$\mathrm{H}$ & 1 & 1.096416 & 2 & 108.376374 & 3 & 170.047537 \\
$\mathrm{H}$ & 6 & 1.097560 & 5 & 108.468111 & 4 & 57.948062 \\
$\mathrm{H}$ & 6 & 1.093660 & 5 & 107.853646 & 4 & 174.865263 \\
$\mathrm{H}$ & 3 & 1.088359 & 2 & 117.086167 & 1 & -41.501719 \\
$\mathrm{H}$ & 4 & 1.025610 & 3 & 100.066453 & 2 & -165.618289 \\
$\mathrm{H}$ & 7 & 1.083957 & 3 & 123.687111 & 2 & -14.010986
\end{tabular}

\section{$23(\mathrm{R}=\mathrm{Cl}) \mathrm{TS}$ (7-endo)}

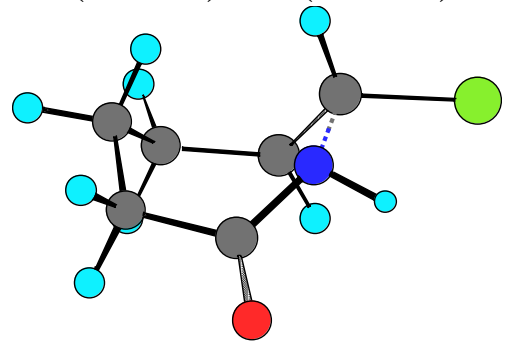

$\%$ chk=23-Cl-7-endo

\# ub3lyp/6-31 $\mathrm{g} * \mathrm{opt}=(\mathrm{ts}$, noeigen,readfc,nofreeze $)$ freq=noraman

calculate for 23-Cl-7-endo $\quad \mathrm{HF}=-824.138248$

\begin{tabular}{lllllll}
02 & \multicolumn{7}{l}{$l$} & & & \\
$\mathrm{C}$ & & & & & & \\
$\mathrm{C}$ & 1 & 1.506365 & & & & \\
$\mathrm{~N}$ & 1 & 2.752114 & 2 & 99.774560 & & \\
$\mathrm{C}$ & 2 & 1.541594 & 1 & 114.607501 & 3 & 12.259836 \\
$\mathrm{C}$ & 3 & 1.375964 & 1 & 90.693318 & 2 & 47.415845 \\
$\mathrm{C}$ & 5 & 1.527575 & 3 & 118.512176 & 1 & -58.208744 \\
$\mathrm{O}$ & 5 & 1.232106 & 3 & 120.993650 & 1 & 129.294479 \\
$\mathrm{C}$ & 1 & 1.367671 & 2 & 121.875952 & 4 & -36.500288 \\
$\mathrm{Cl}$ & 8 & 1.755868 & 1 & 120.356334 & 2 & -174.525506 \\
$\mathrm{H}$ & 2 & 1.097099 & 1 & 108.808043 & 8 & -159.276458 \\
$\mathrm{H}$ & 4 & 1.096094 & 2 & 110.372960 & 1 & 58.051155 \\
$\mathrm{H}$ & 2 & 1.101856 & 1 & 109.007811 & 8 & 85.930037 \\
$\mathrm{H}$ & 4 & 1.096824 & 2 & 108.052356 & 1 & 173.591183 \\
$\mathrm{H}$ & 6 & 1.098566 & 5 & 105.942042 & 3 & -101.177170 \\
$\mathrm{H}$ & 6 & 1.096532 & 5 & 106.407703 & 3 & 146.715944 \\
$\mathrm{H}$ & 3 & 1.025257 & 1 & 107.411625 & 2 & 156.791347 \\
$\mathrm{H}$ & 1 & 1.086557 & 2 & 119.185689 & 4 & 138.320861 \\
$\mathrm{H}$ & 8 & 1.080320 & 1 & 123.584941 & 2 & -18.929087
\end{tabular}




\section{$23(\mathrm{R}=\mathrm{Cl}) \mathrm{TS}(1,5-\mathrm{H}$ migration $)$}

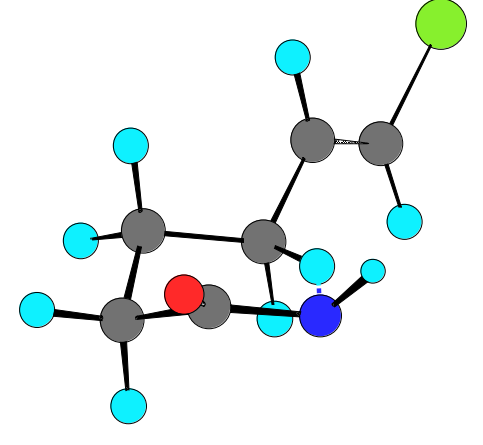

\%chk=23-Cl-1,5-H

\# ub31yp/6-31g* opt=(ts,noeigen,readfc,nofreeze) freq=noraman

calculate for $23-\mathrm{Cl}-1,5-\mathrm{H} \quad \mathrm{HF}=-824.136224$

$\begin{array}{ll}02 & 2 \\ \mathrm{C} & \mathrm{C} \\ \mathrm{C} \\ \mathrm{O} \\ \mathrm{N} \\ \mathrm{C} \\ \mathrm{C} \\ \mathrm{C} \\ \mathrm{Cl} \\ \mathrm{H} \\ \mathrm{H} \\ \mathrm{H} \\ \mathrm{H} \\ \mathrm{H} \\ \mathrm{H} \\ \mathrm{H} \\ \mathrm{H} \\ \mathrm{H}\end{array}$

$\begin{array}{lccccc}1 & 2.517688 & & & & \\ 2 & 1.524017 & 1 & 35.108024 & & \\ 2 & 1.228707 & 1 & 137.213087 & 3 & -83.590282 \\ 2 & 1.379759 & 1 & 92.629130 & 3 & 130.061703 \\ 1 & 1.533333 & 2 & 90.685845 & 4 & 147.960990 \\ 6 & 1.469614 & 1 & 117.220950 & 2 & -127.355150 \\ 7 & 1.342115 & 6 & 123.274180 & 1 & -144.292891 \\ 8 & 1.742250 & 7 & 123.134062 & 6 & -178.416403 \\ 3 & 1.092522 & 2 & 108.246231 & 1 & -122.043553 \\ 3 & 1.097883 & 2 & 108.939754 & 1 & 120.515128 \\ 1 & 1.097492 & 2 & 144.908445 & 4 & -83.786407 \\ 6 & 1.225537 & 1 & 101.366129 & 2 & -7.385064 \\ 6 & 1.096614 & 1 & 112.477155 & 2 & 99.195715 \\ 1 & 1.098257 & 2 & 91.676164 & 4 & 38.621991 \\ 7 & 1.088983 & 6 & 117.703712 & 1 & 37.230022 \\ 8 & 1.084687 & 7 & 123.821757 & 6 & 1.668099 \\ 5 & 1.025186 & 2 & 111.000939 & 1 & 123.962803\end{array}$

$22(\mathrm{R}=\mathrm{Me})$-radical

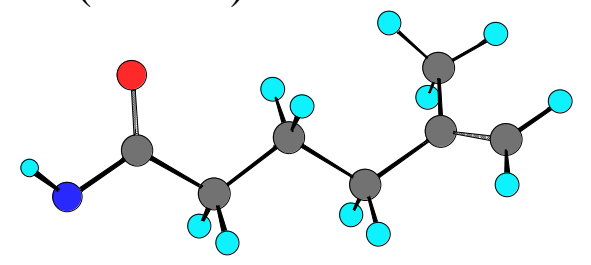

$\%$ chk=22 $(\mathbf{R}=\mathbf{M e})$-radical

\# ub3lyp/6-31g* opt freq=noraman

calculate for $22(\mathrm{R}=\mathrm{Me})$-radical $\mathrm{HF}=-403.869303$

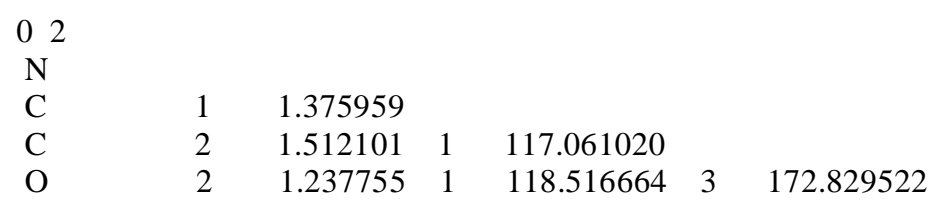




$\begin{array}{lllllll}\mathrm{C} & 3 & 1.530316 & 2 & 113.734154 & 1 & 178.635214 \\ \mathrm{C} & 5 & 1.541152 & 3 & 112.086488 & 2 & -179.860666 \\ \mathrm{C} & 6 & 1.515182 & 5 & 113.896786 & 3 & -178.920314 \\ \mathrm{C} & 7 & 1.337042 & 6 & 121.372825 & 5 & 113.235100 \\ \mathrm{C} & 7 & 1.509973 & 6 & 116.848425 & 5 & -67.110600 \\ \mathrm{H} & 3 & 1.098868 & 2 & 107.464973 & 1 & 54.981940 \\ \mathrm{H} & 1 & 1.028581 & 2 & 108.361816 & 4 & -30.666063 \\ \mathrm{H} & 3 & 1.100059 & 2 & 107.567637 & 1 & -57.739908 \\ \mathrm{H} & 5 & 1.096893 & 3 & 109.662108 & 2 & 58.409285 \\ \mathrm{H} & 5 & 1.095310 & 3 & 108.806106 & 2 & -57.218946 \\ \mathrm{H} & 6 & 1.097599 & 5 & 109.116259 & 3 & -56.531062 \\ \mathrm{H} & 6 & 1.101458 & 5 & 108.576065 & 3 & 59.141236 \\ \mathrm{H} & 8 & 1.087414 & 7 & 121.783367 & 6 & -179.979546 \\ \mathrm{H} & 8 & 1.087536 & 7 & 121.769310 & 6 & 0.093568 \\ \mathrm{H} & 9 & 1.094171 & 7 & 111.534588 & 6 & -177.492207 \\ \mathrm{H} & 9 & 1.098933 & 7 & 110.971303 & 6 & -56.913595 \\ \mathrm{H} & 9 & 1.097665 & 7 & 111.711448 & 6 & 61.803426\end{array}$

\section{$22(\mathrm{R}=\mathrm{Me}), \mathrm{TS}(6-\mathrm{exo})$}

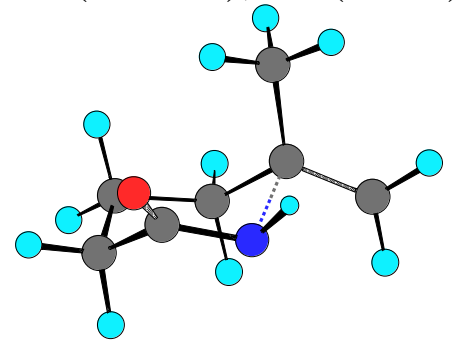

$\%$ chk=22-Me-6-exo

\# ub3lyp/6-31g* opt=(ts,noeigen,readfc,nofreeze) freq=noraman

calculate for 22-Me-6-exo $\quad \mathrm{HF}=-403.861359$

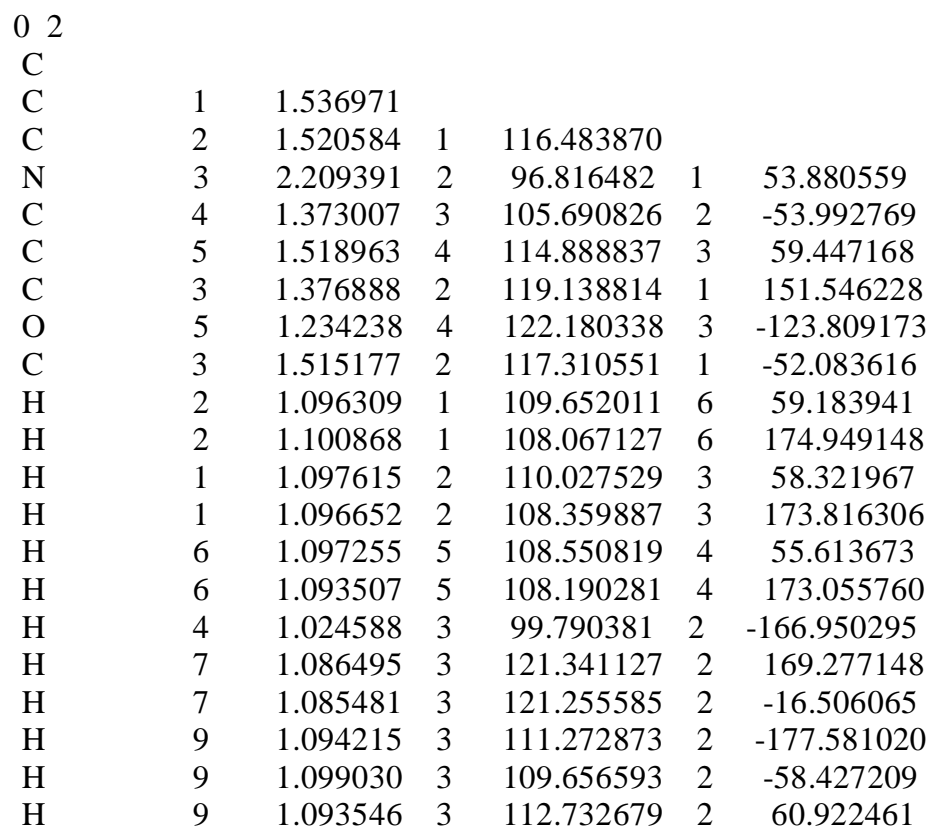

$22(\mathrm{R}=\mathrm{Me}) \mathrm{TS}(7$-endo $)$ 


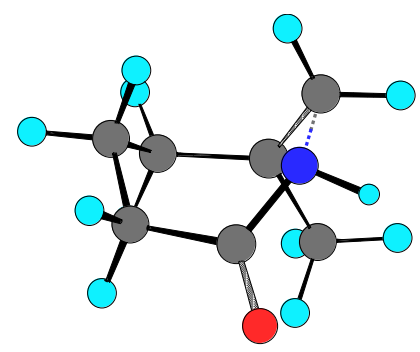

$\%$ chk=22-Me-7-endo

\# ub31yp/6-31 g* opt=(ts,noeigen,readfc,nofreeze) freq=noraman

calculate for 22-Me-7-endo $\mathrm{HF}=-403.865796$

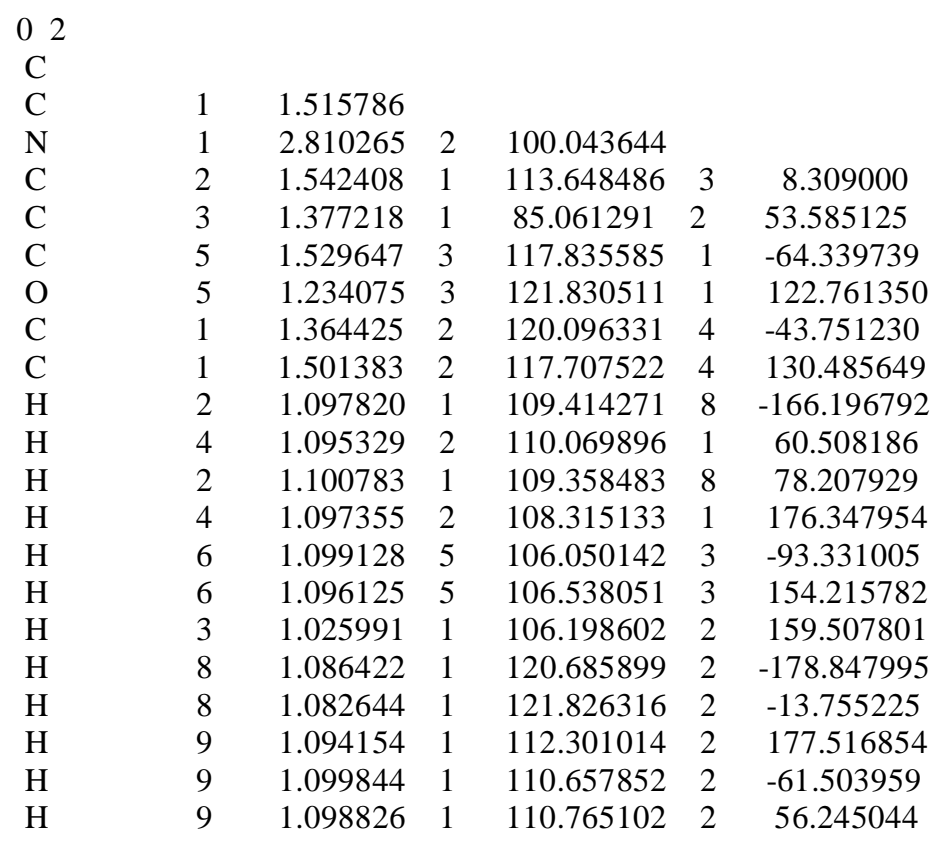

$23(\mathrm{R}=\mathrm{Me})$-radical

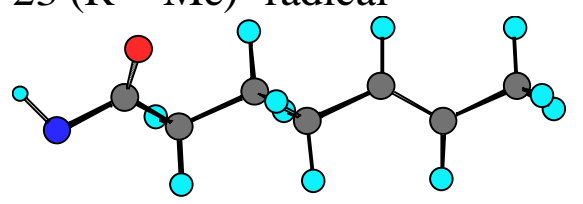

$\%$ chk=23 $(\mathbf{R}=\mathbf{M e})$-radical

\# ub3lyp/6-31g* opt freq=noraman

calculate for $23(\mathrm{R}=\mathrm{Me})$-radical $\mathrm{HF}=-403.870276$

$\begin{array}{llllllr}02 & & & & & & \\ \mathrm{~N} & & & & & & \\ \mathrm{C} & 1 & 1.375864 & & & & \\ \mathrm{C} & 2 & 1.511670 & 1 & 117.167224 & & \\ \mathrm{O} & 2 & 1.237996 & 1 & 118.414771 & 3 & -173.176869 \\ \mathrm{C} & 3 & 1.530295 & 2 & 113.873060 & 1 & -173.564696 \\ \mathrm{C} & 5 & 1.540042 & 3 & 112.389074 & 2 & -178.189707 \\ \mathrm{C} & 6 & 1.504814 & 5 & 112.765872 & 3 & 178.572365 \\ \mathrm{C} & 7 & 1.335596 & 6 & 125.256885 & 5 & 119.062481\end{array}$




$\begin{array}{lcccccc}\mathrm{C} & 8 & 1.502069 & 7 & 125.313550 & 6 & -179.567830 \\ \mathrm{H} & 3 & 1.100819 & 2 & 107.312292 & 1 & 63.229361 \\ \mathrm{H} & 3 & 1.098196 & 2 & 107.645398 & 1 & -49.448085 \\ \mathrm{H} & 5 & 1.095912 & 3 & 109.465842 & 2 & 59.700898 \\ \mathrm{H} & 5 & 1.097486 & 3 & 109.424507 & 2 & -55.940034 \\ \mathrm{H} & 6 & 1.098459 & 5 & 109.695941 & 3 & -58.887145 \\ \mathrm{H} & 6 & 1.101768 & 5 & 108.607549 & 3 & 56.948844 \\ \mathrm{H} & 8 & 1.092125 & 7 & 118.645675 & 6 & 0.604446 \\ \mathrm{H} & 7 & 1.092612 & 6 & 115.900179 & 5 & -60.173411 \\ \mathrm{H} & 9 & 1.098544 & 8 & 111.264237 & 7 & 120.877363 \\ \mathrm{H} & 9 & 1.098603 & 8 & 111.307638 & 7 & -120.463573 \\ \mathrm{H} & 9 & 1.095331 & 8 & 111.552327 & 7 & 0.229366 \\ \mathrm{H} & 1 & 1.028586 & 2 & 108.337089 & 4 & 30.725501\end{array}$

\section{$23(\mathrm{R}=\mathrm{Me}), \mathrm{TS}(6-\mathrm{exo})$}

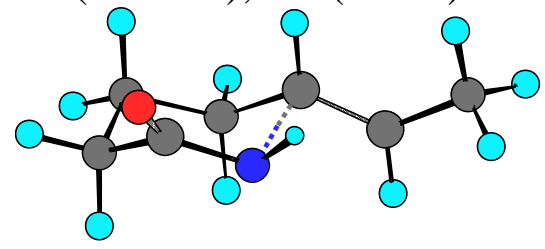

\%chk=23-Me-6-exo

\# ub3lyp/6-31g* opt=(ts,noeigen,readfc,nofreeze) freq=noraman

calculate for 23-Me-6-exo $\quad \mathrm{HF}=-403.866641$

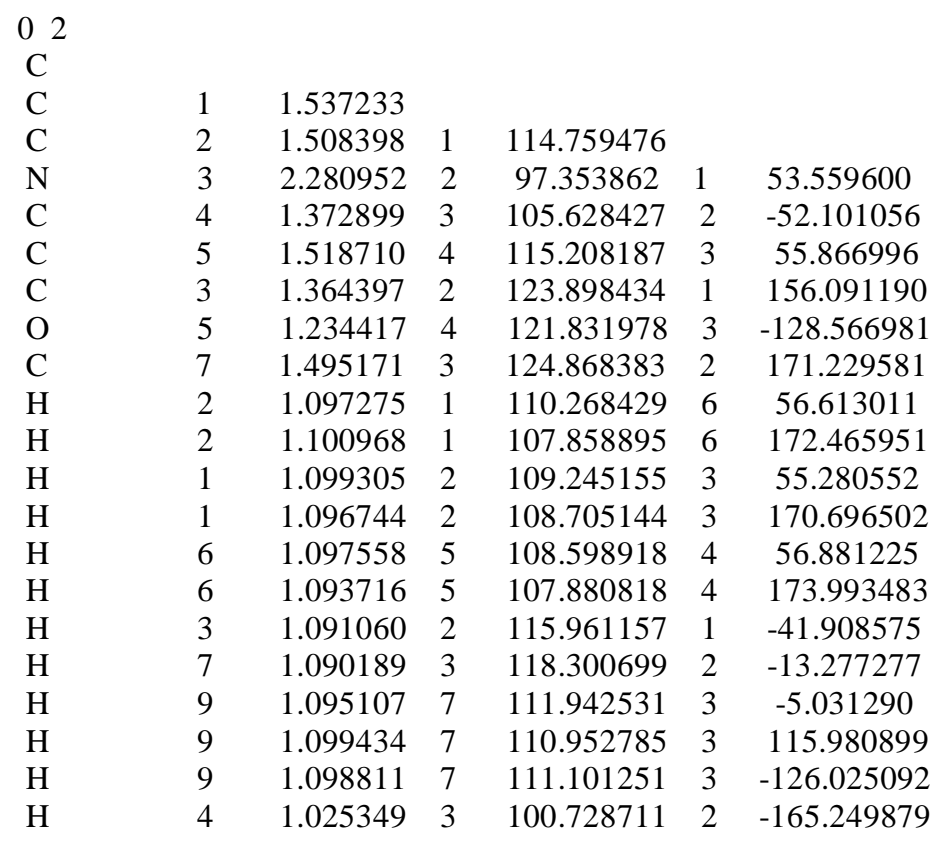

\section{$23(\mathrm{R}=\mathrm{Me}), \mathrm{TS}$ (7-endo)}




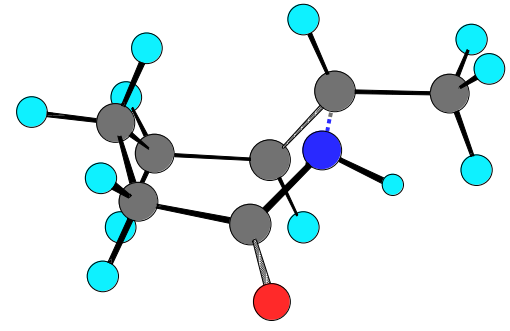

$\%$ chk=23-Me-7-endo

\# ub3lyp/6-31g* opt=(ts,noeigen,readfc,nofreeze) freq=noraman

calculate for 23-Me-7-endo $\quad \mathrm{HF}=-403.864116$

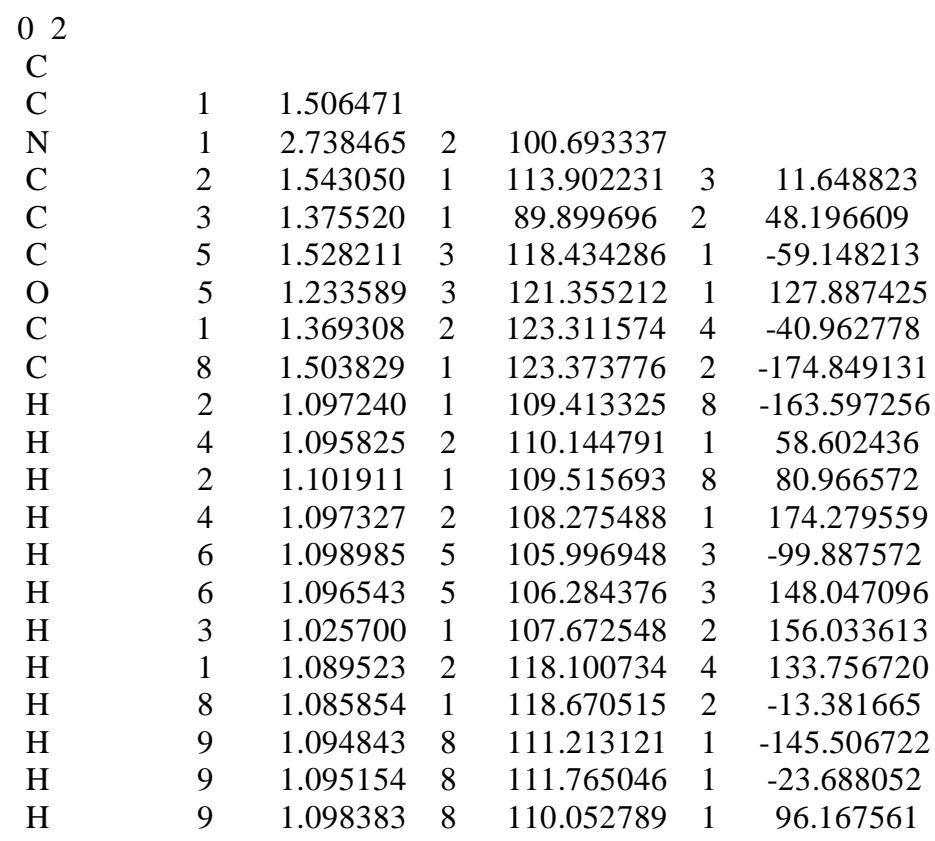


Compound 6e:

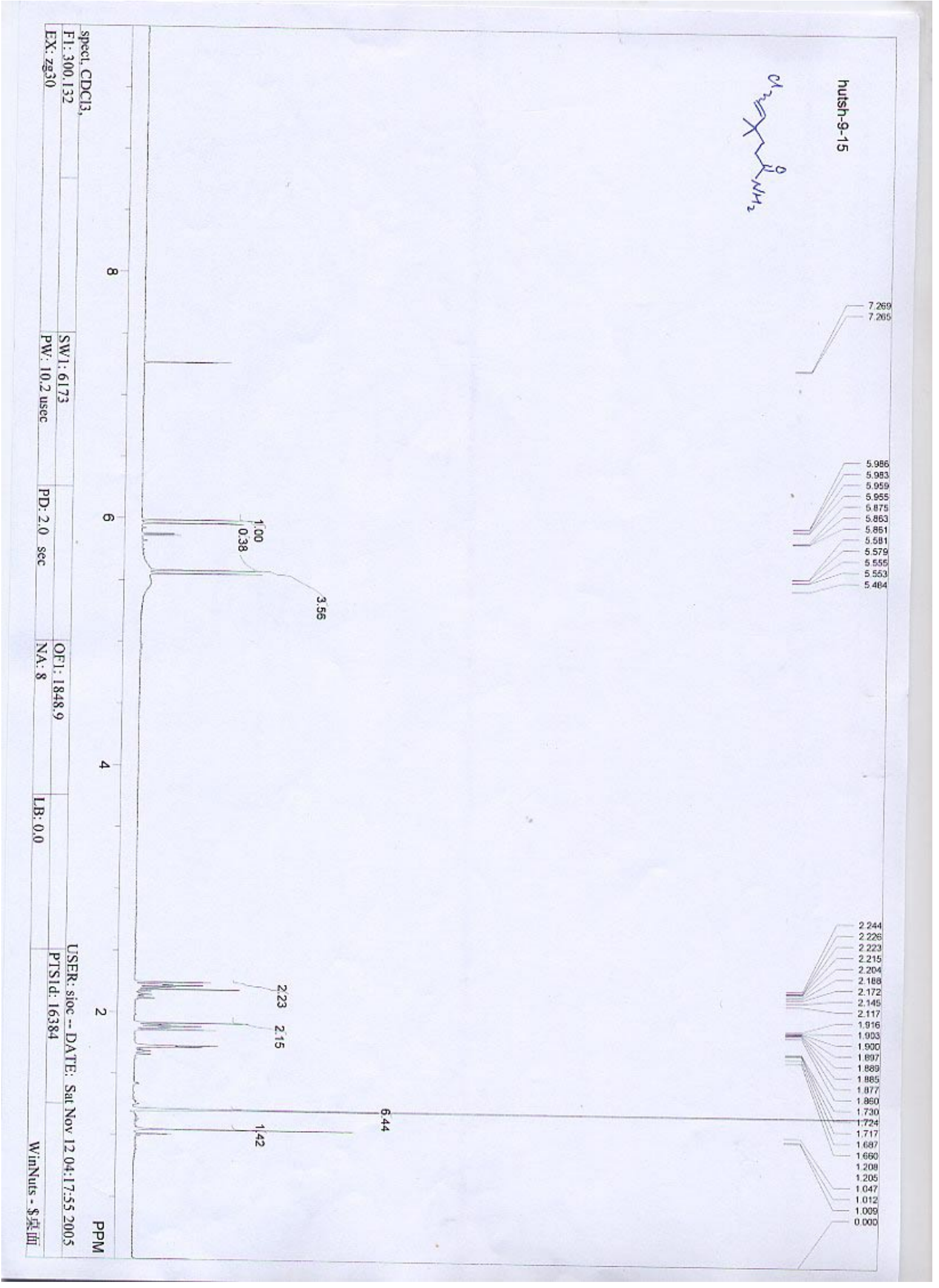


Compound 7a:

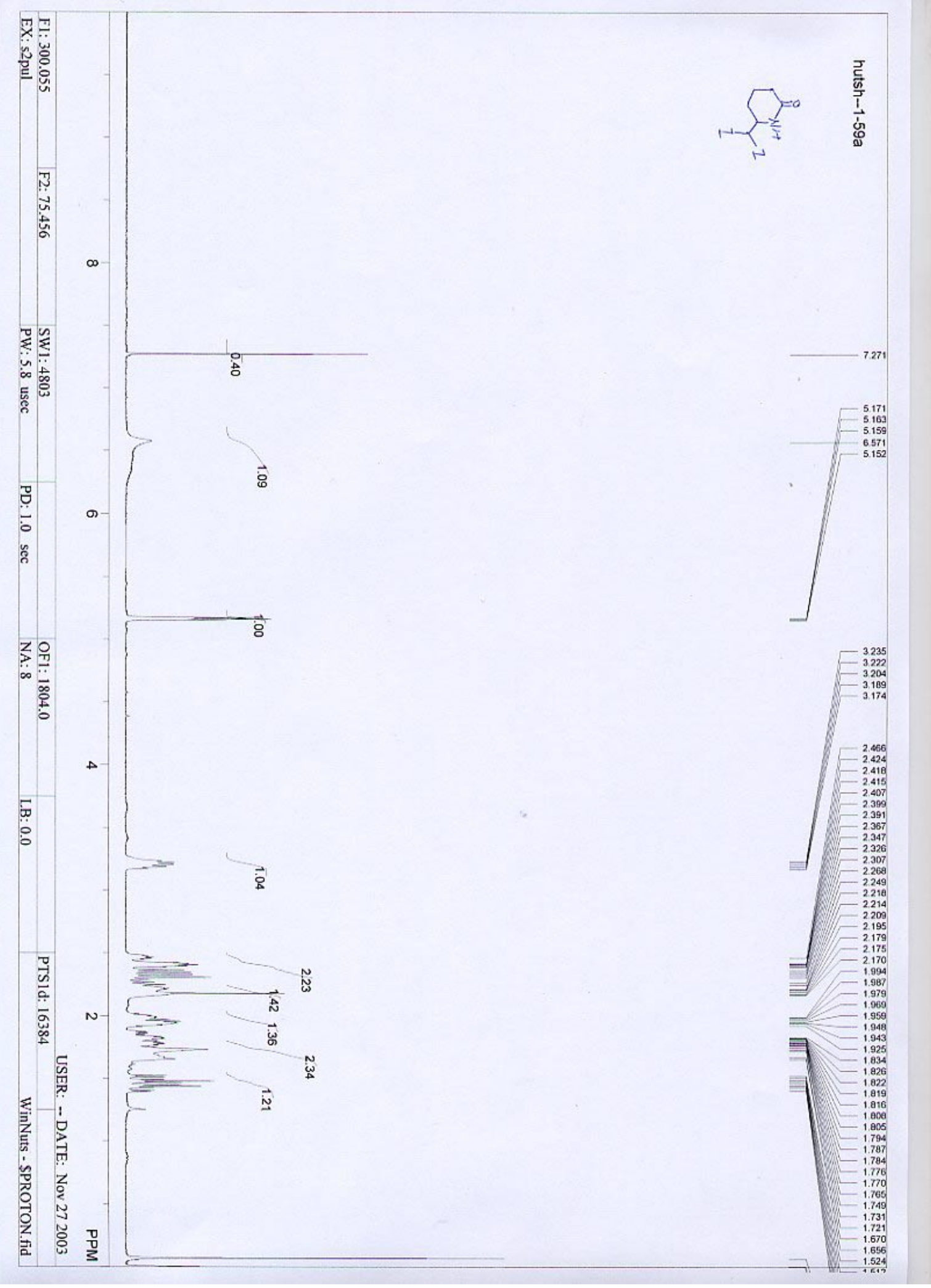




\section{Compound 7d:}

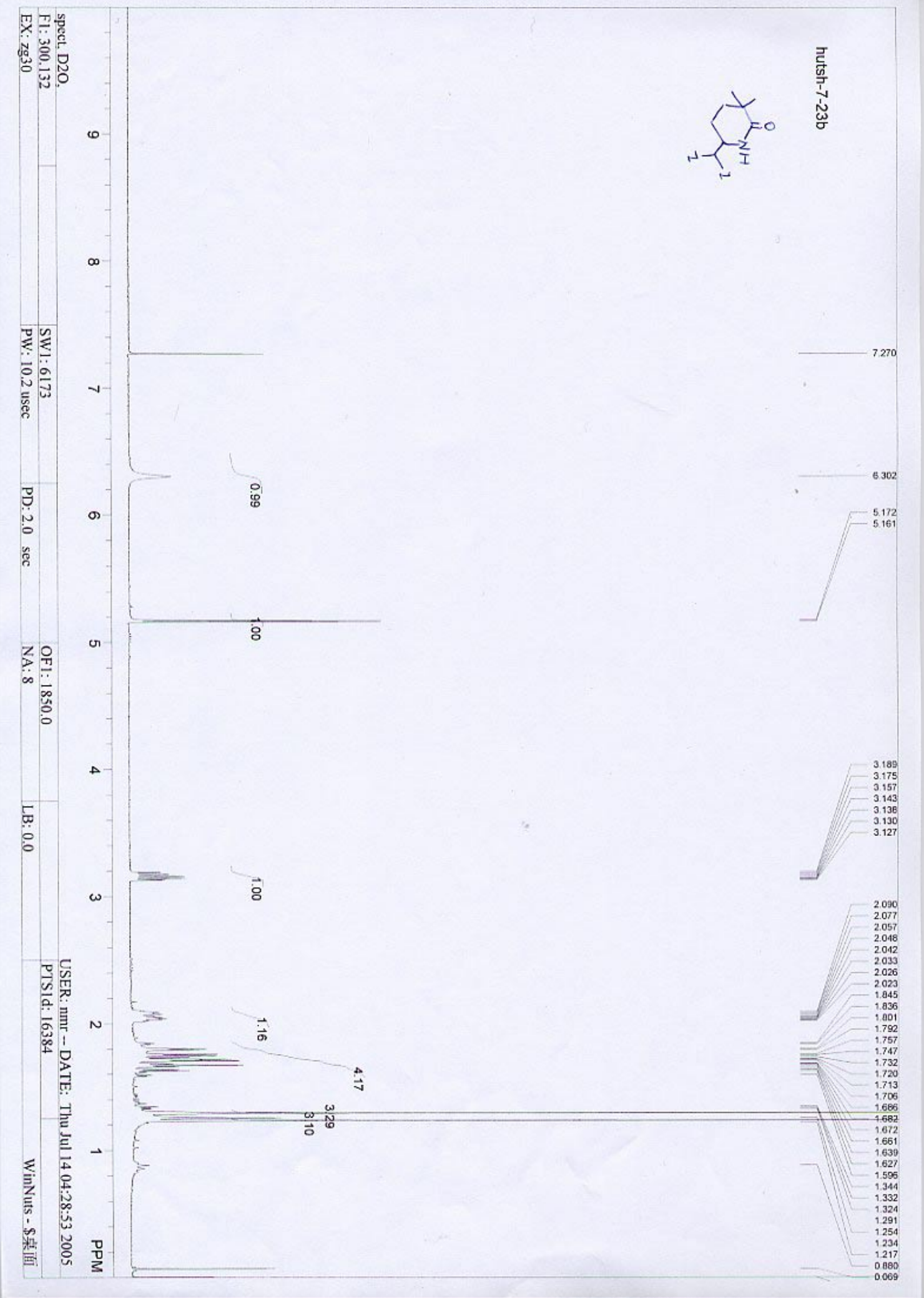


Compound 8e:

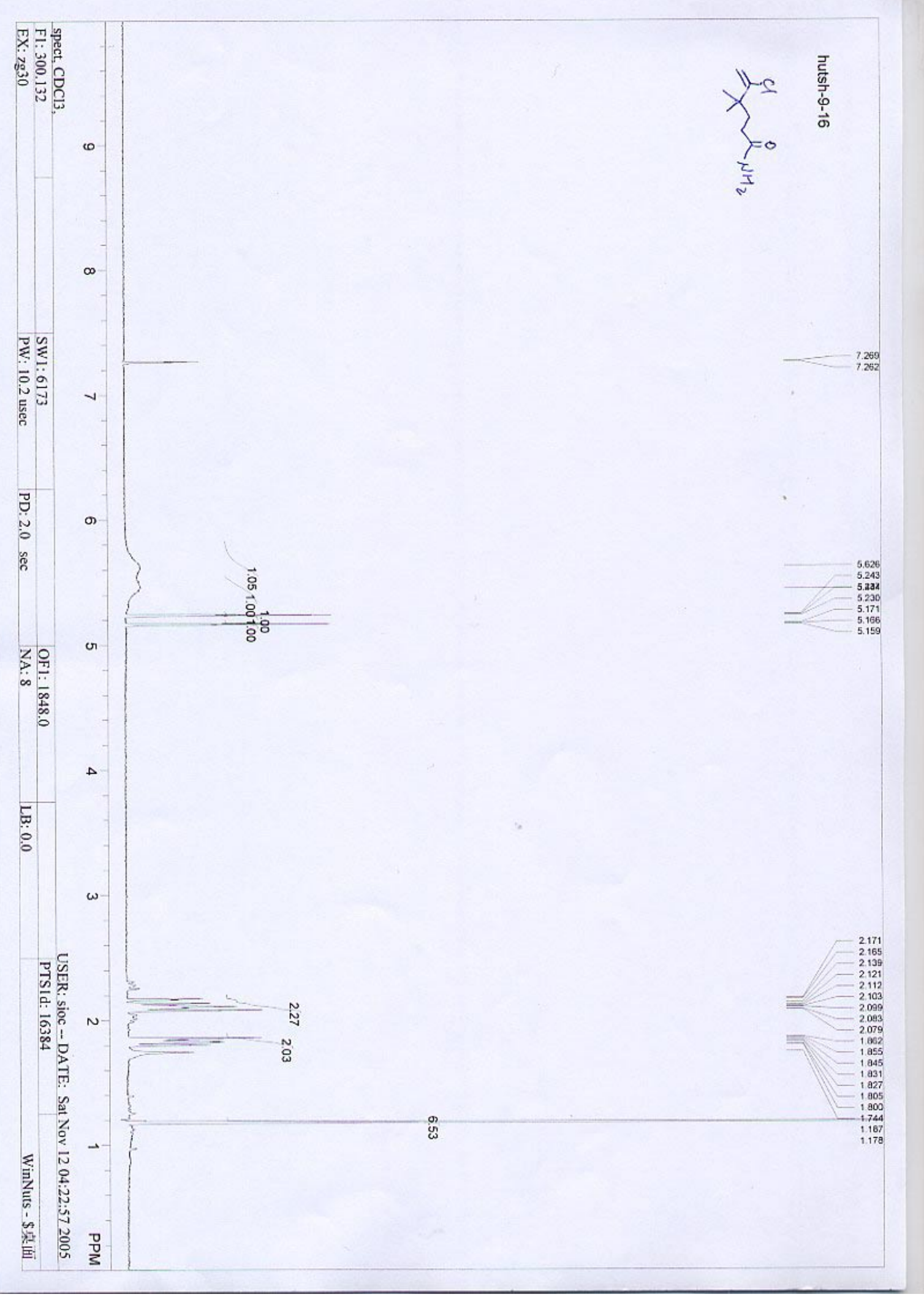


Compound 9a:

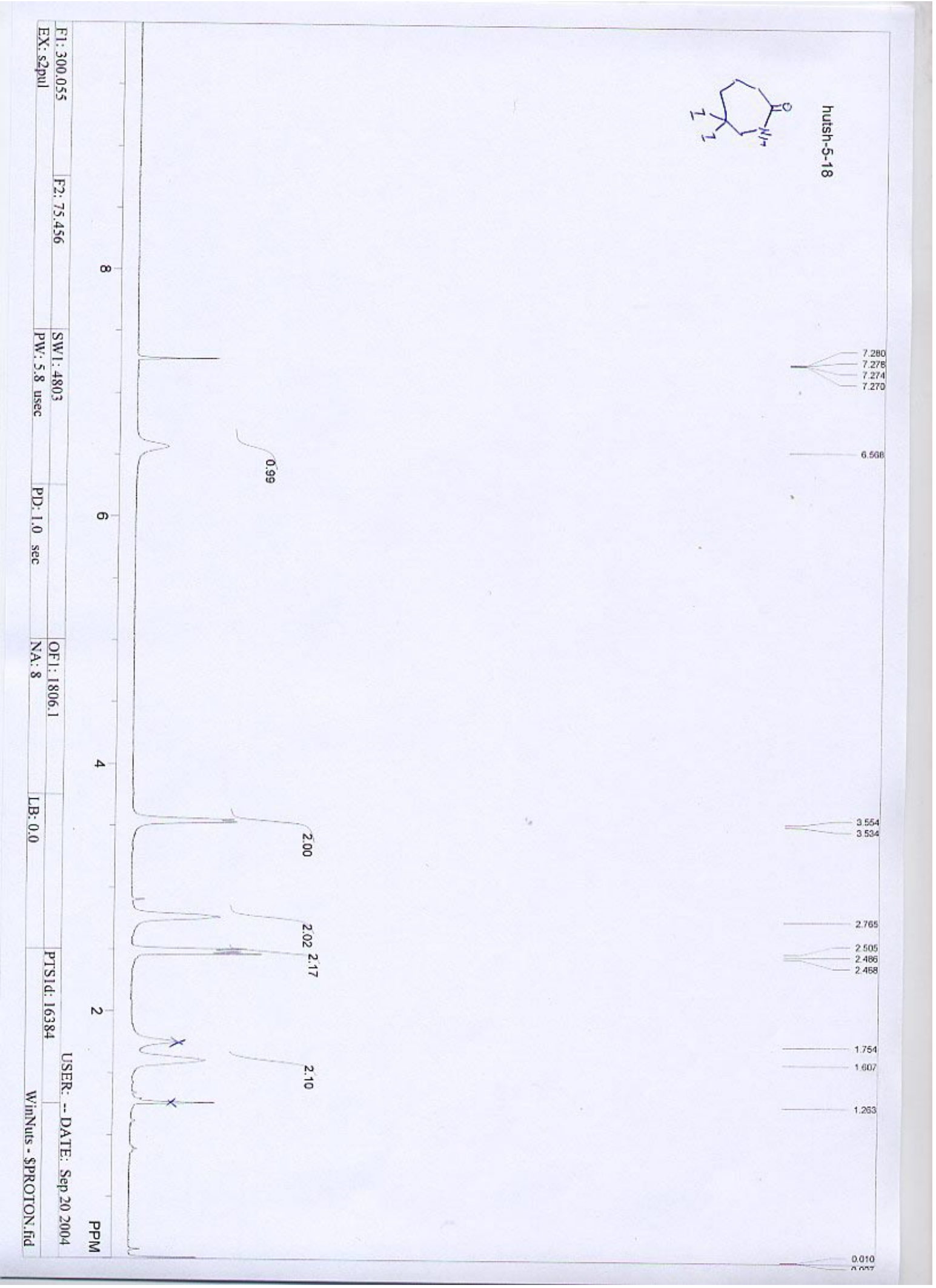


Compound 9d:

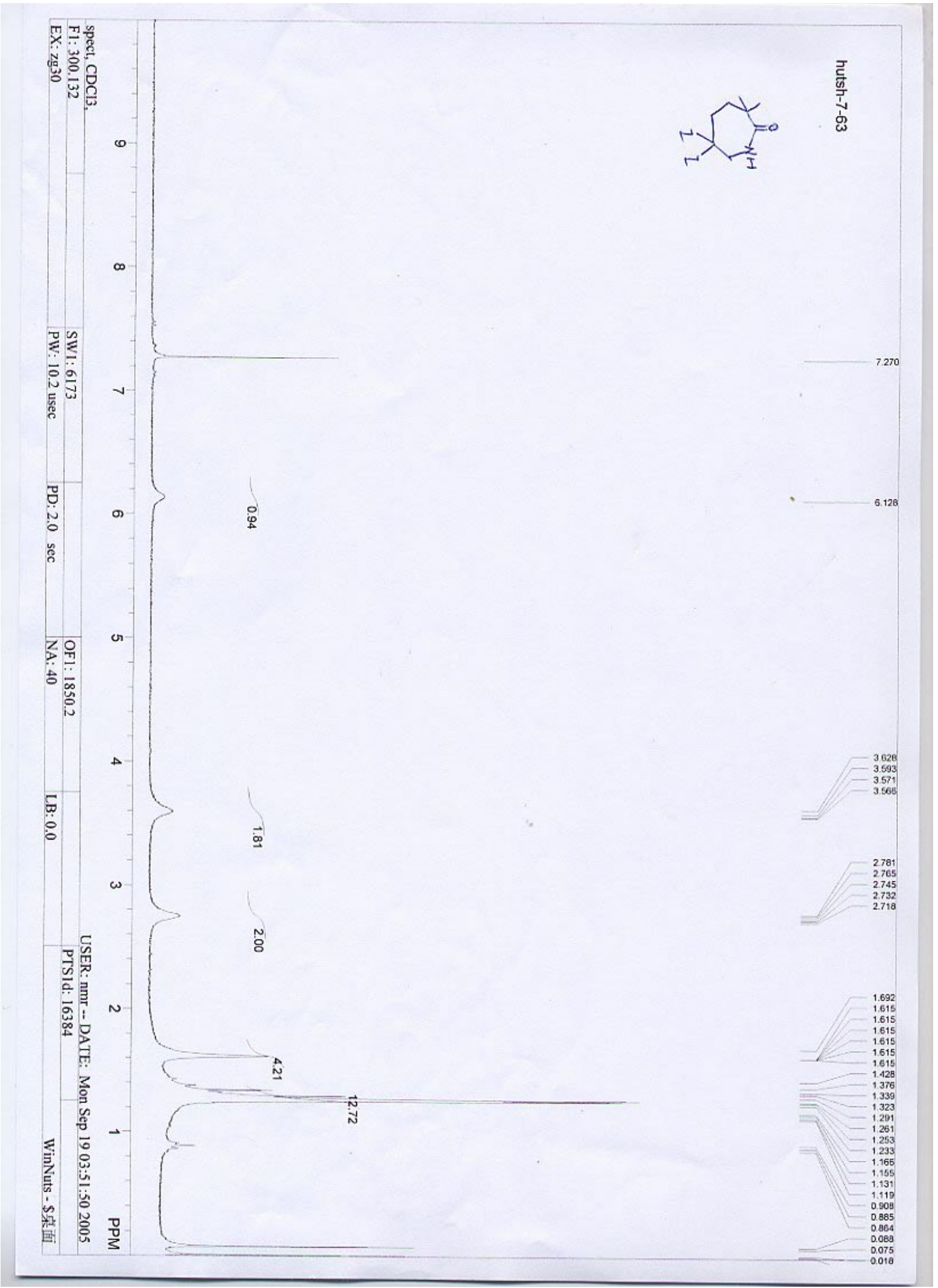


Compound 16a:

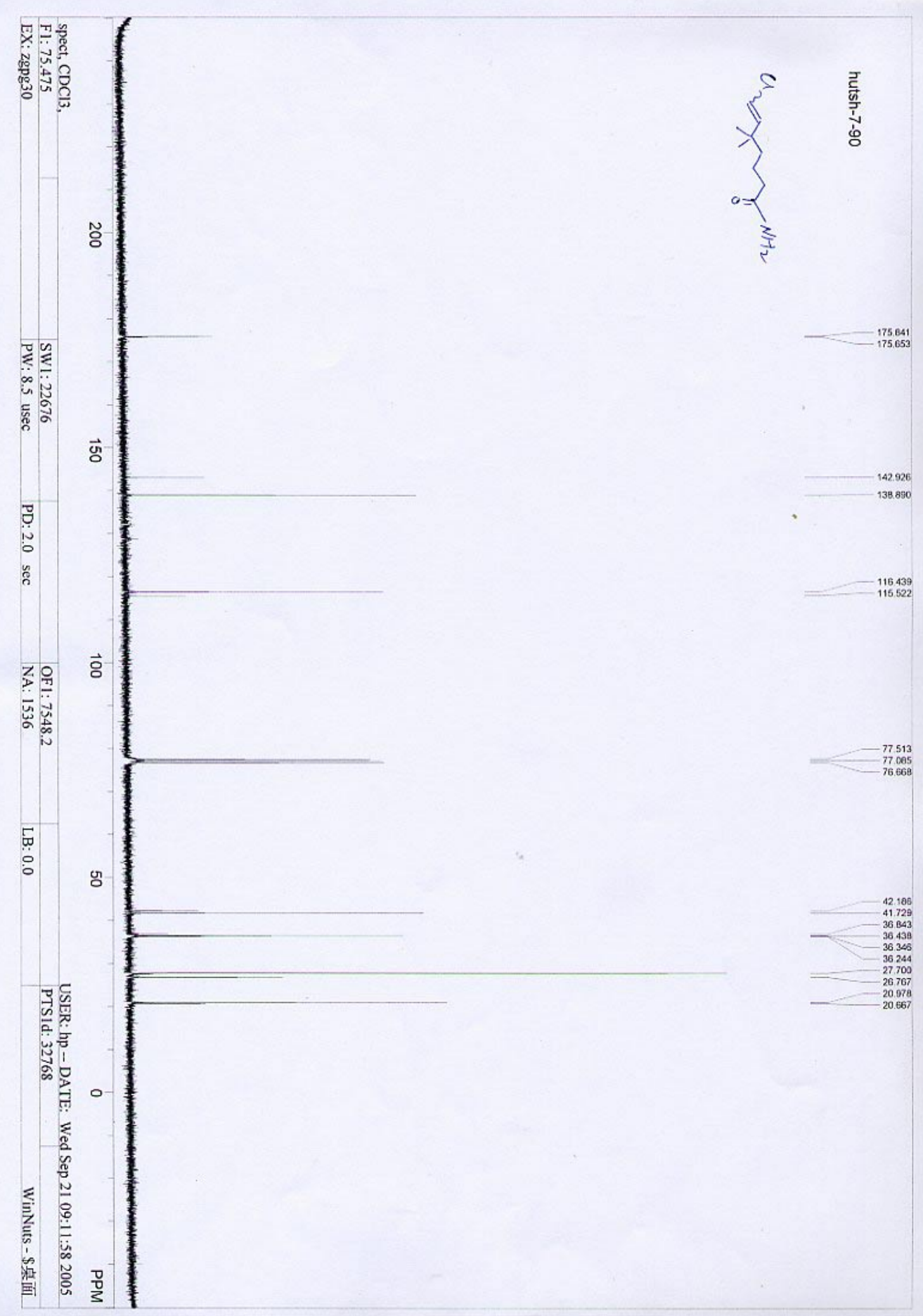


Compound 16c:

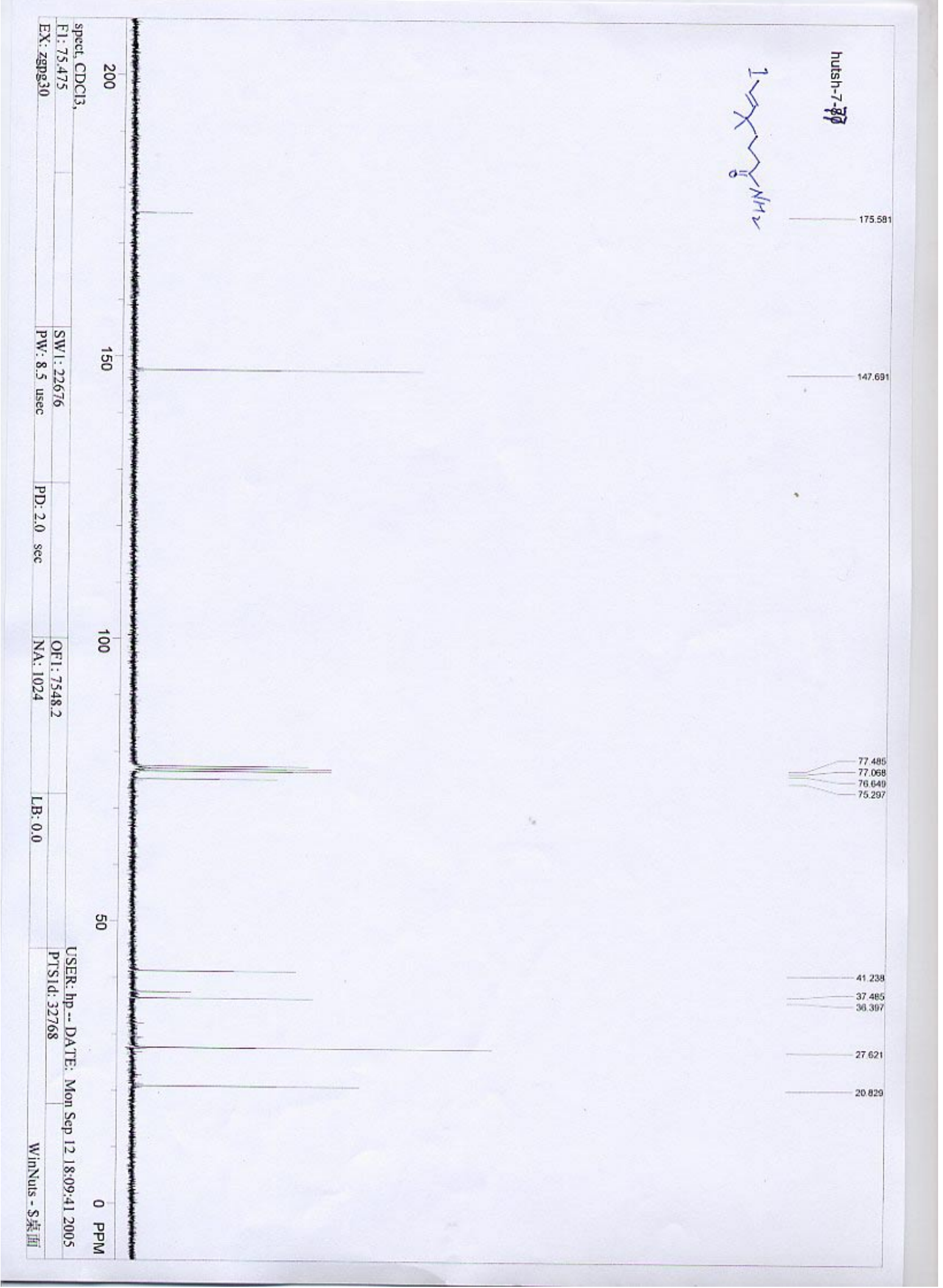


Compound 17a:

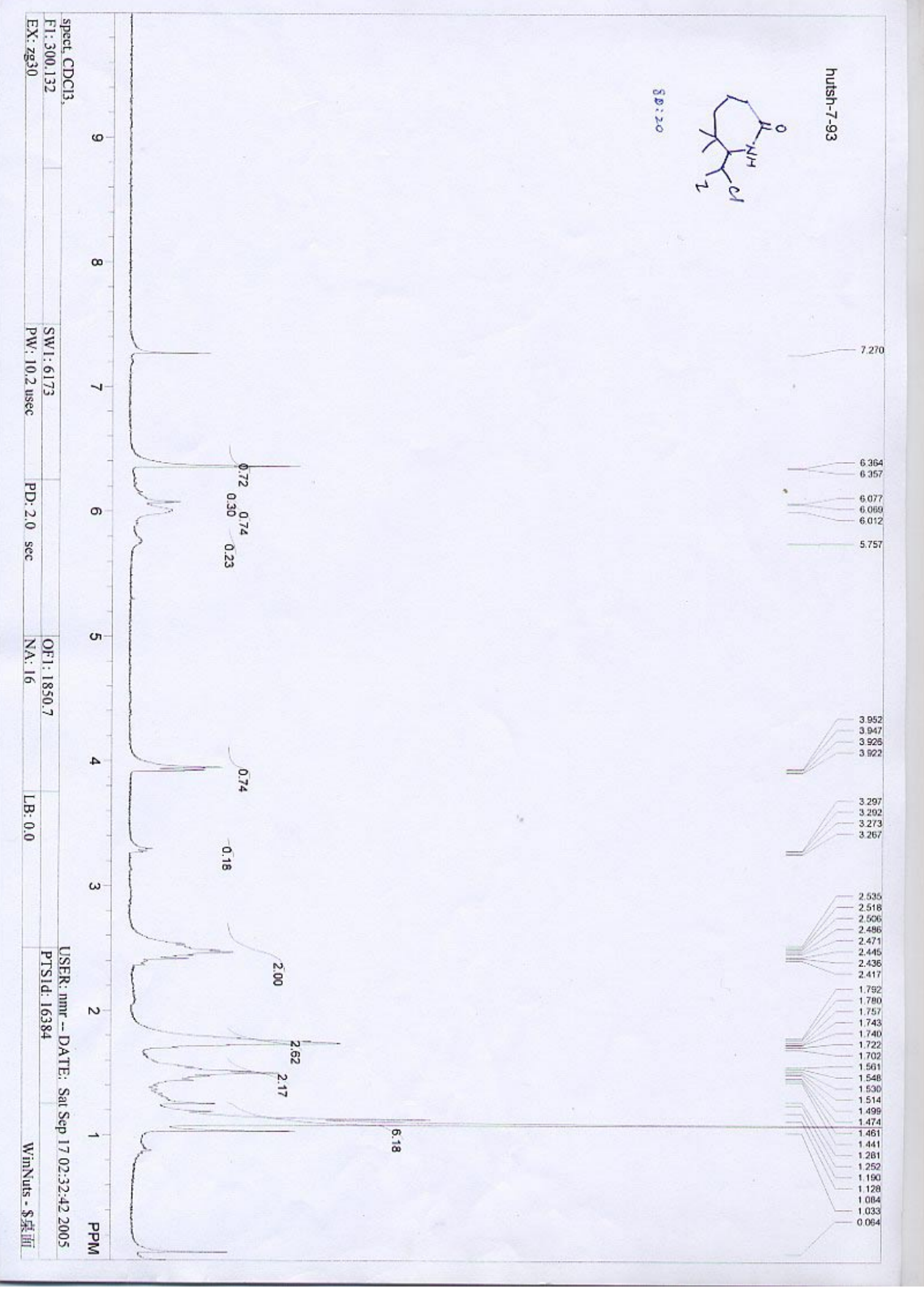


Compound 17b:

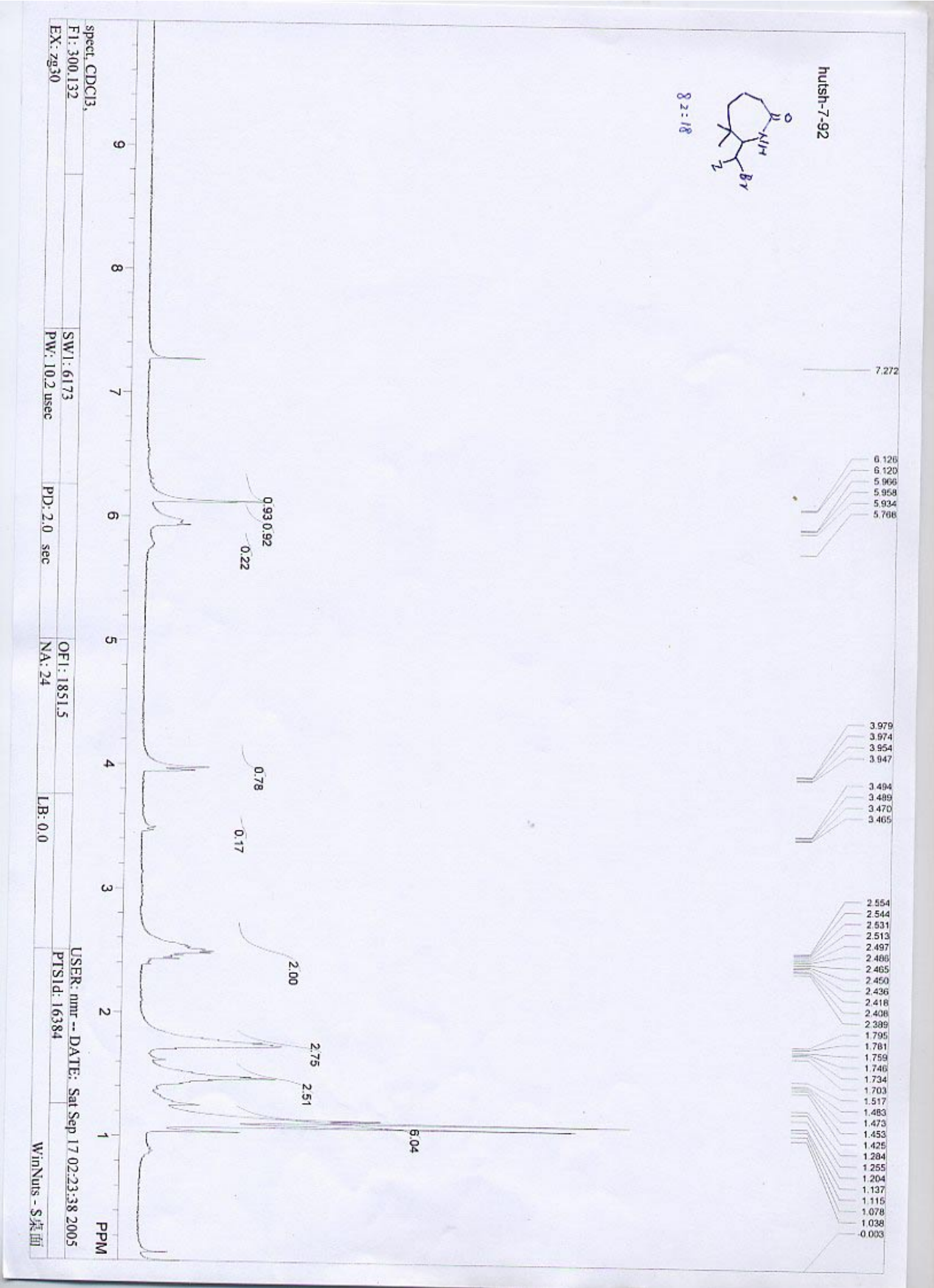


Compound 17c:

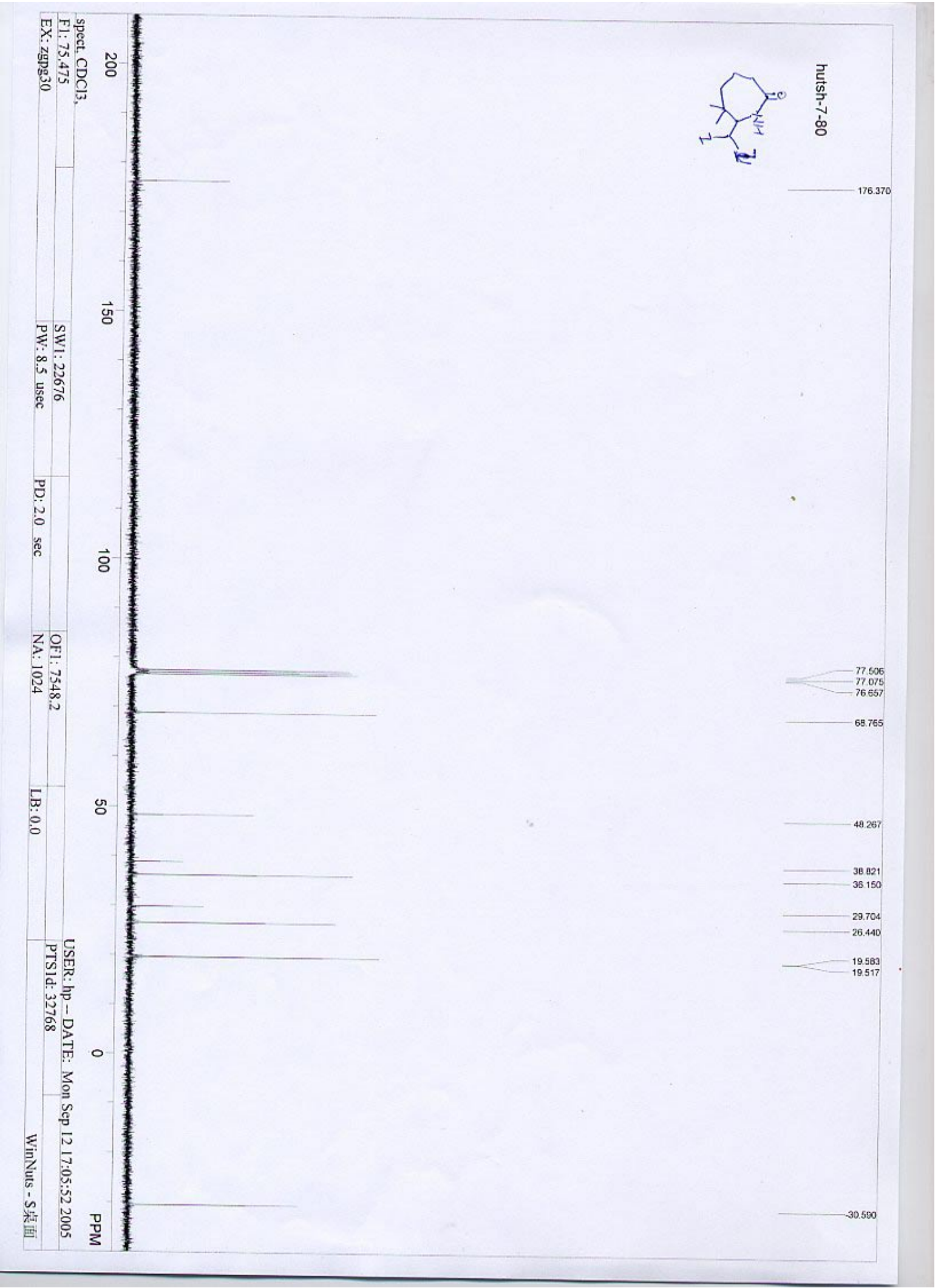


Compound 18a:

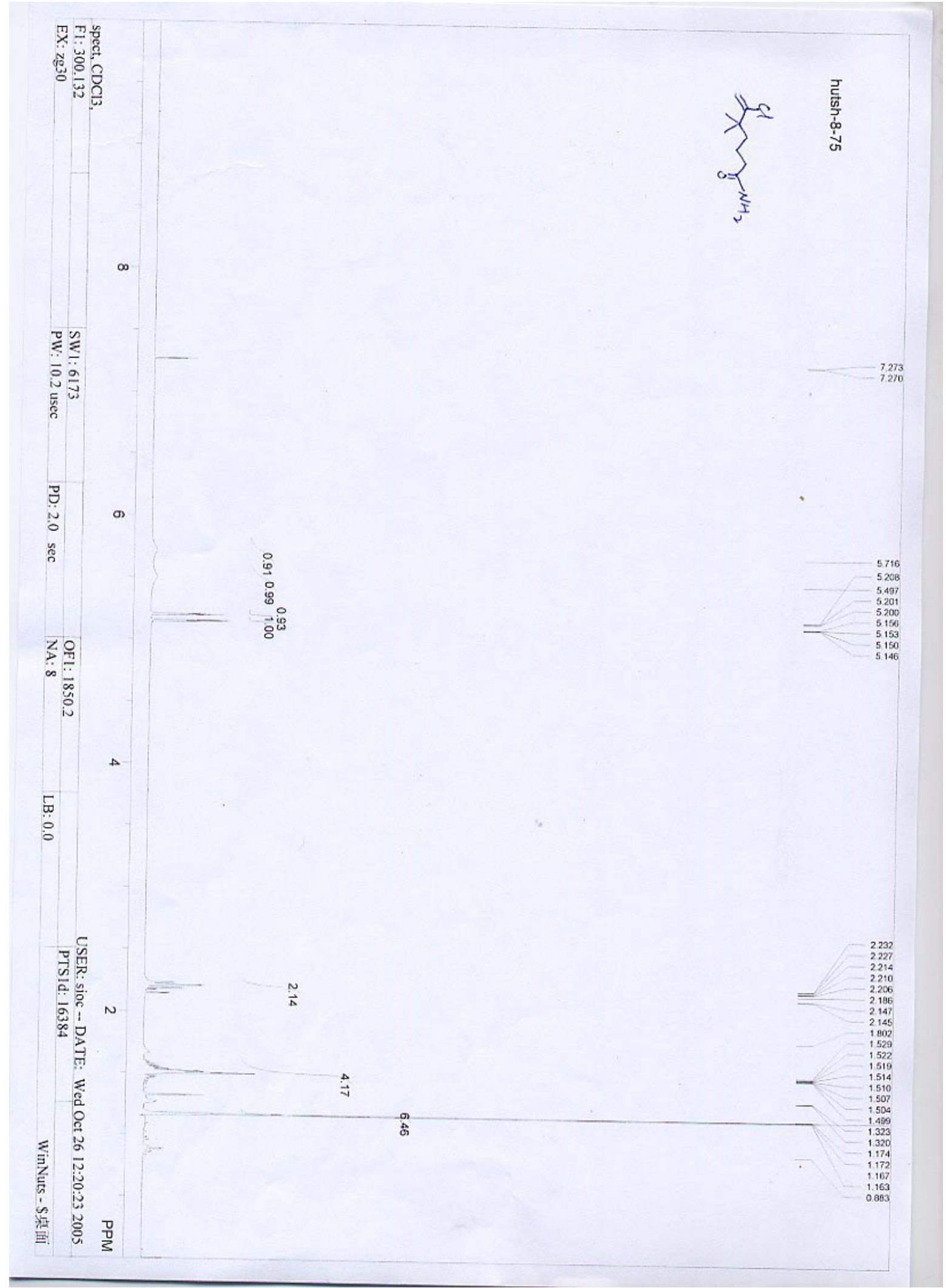


Compound 18b:
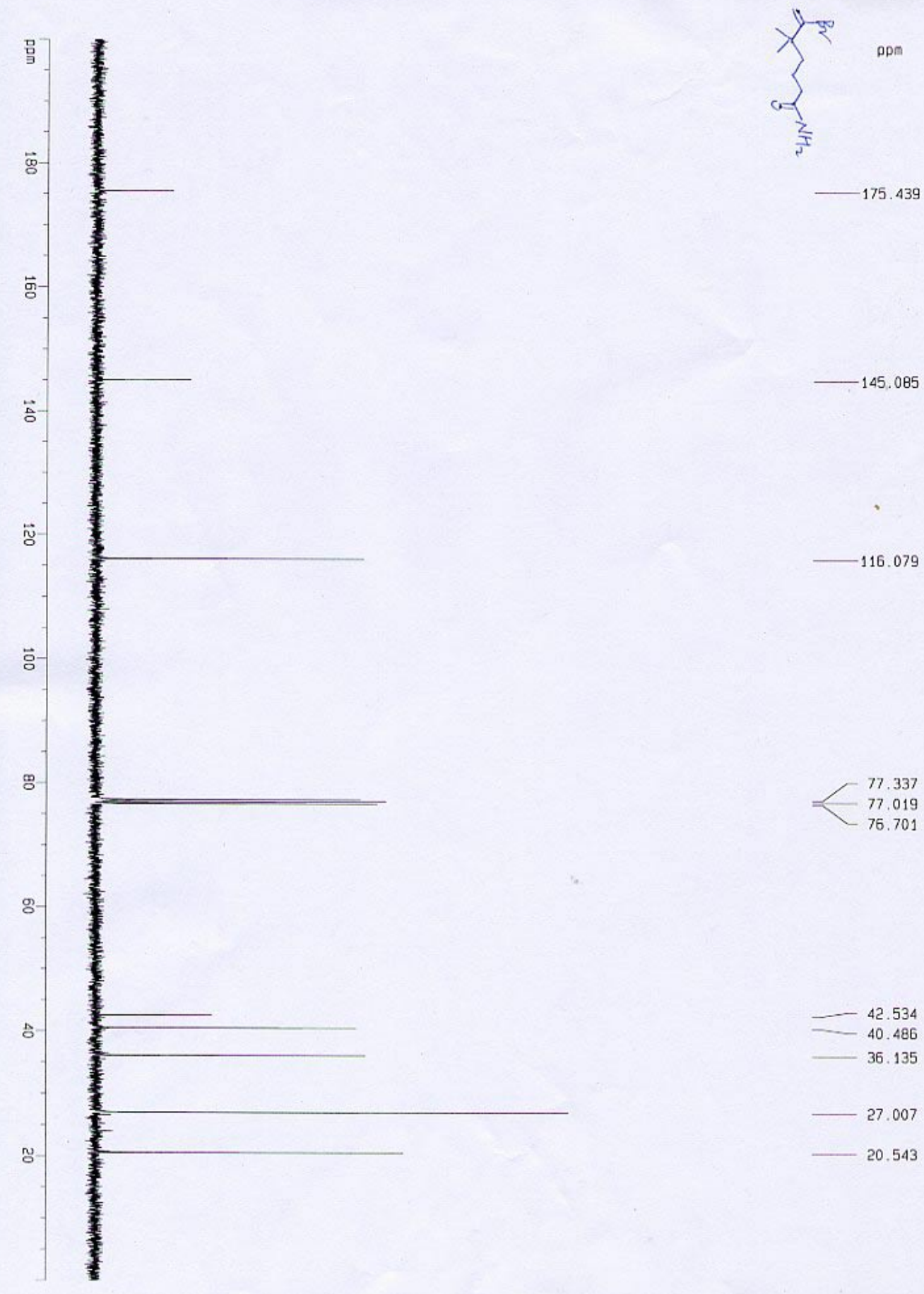

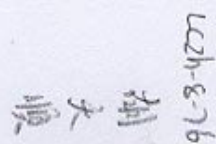


Compound 18c:

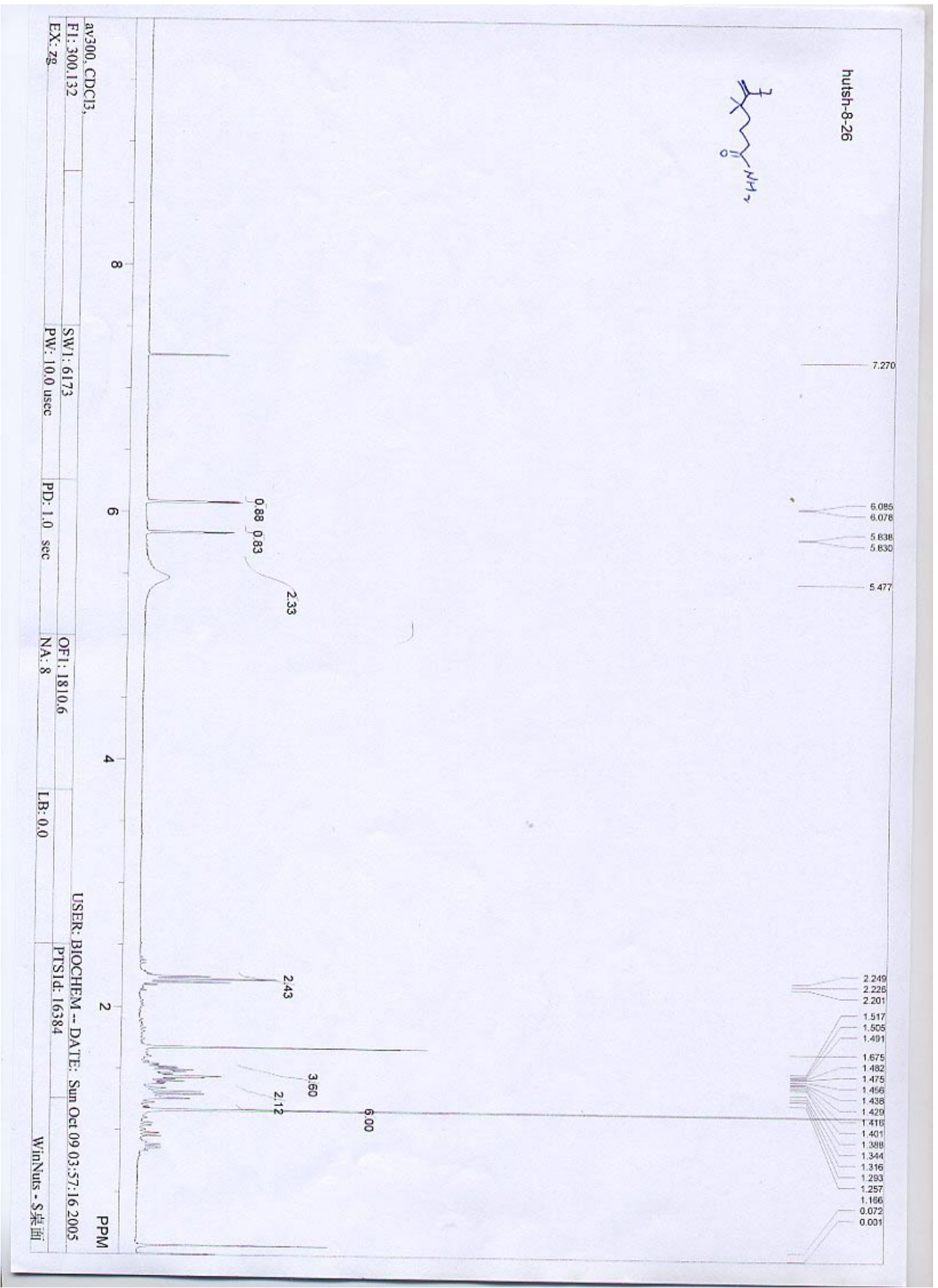


Compound 19a:

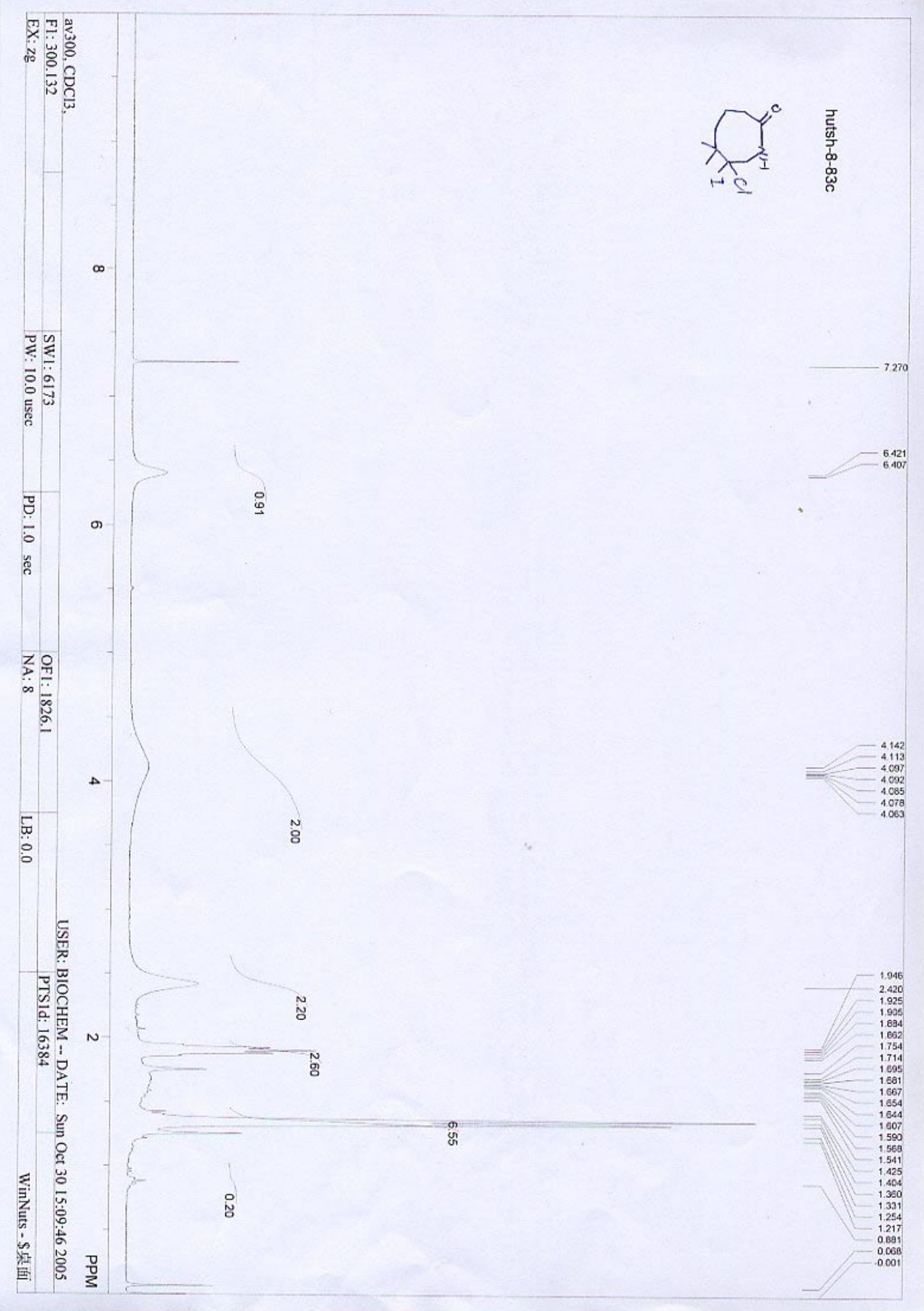




\section{Compound 19b:}

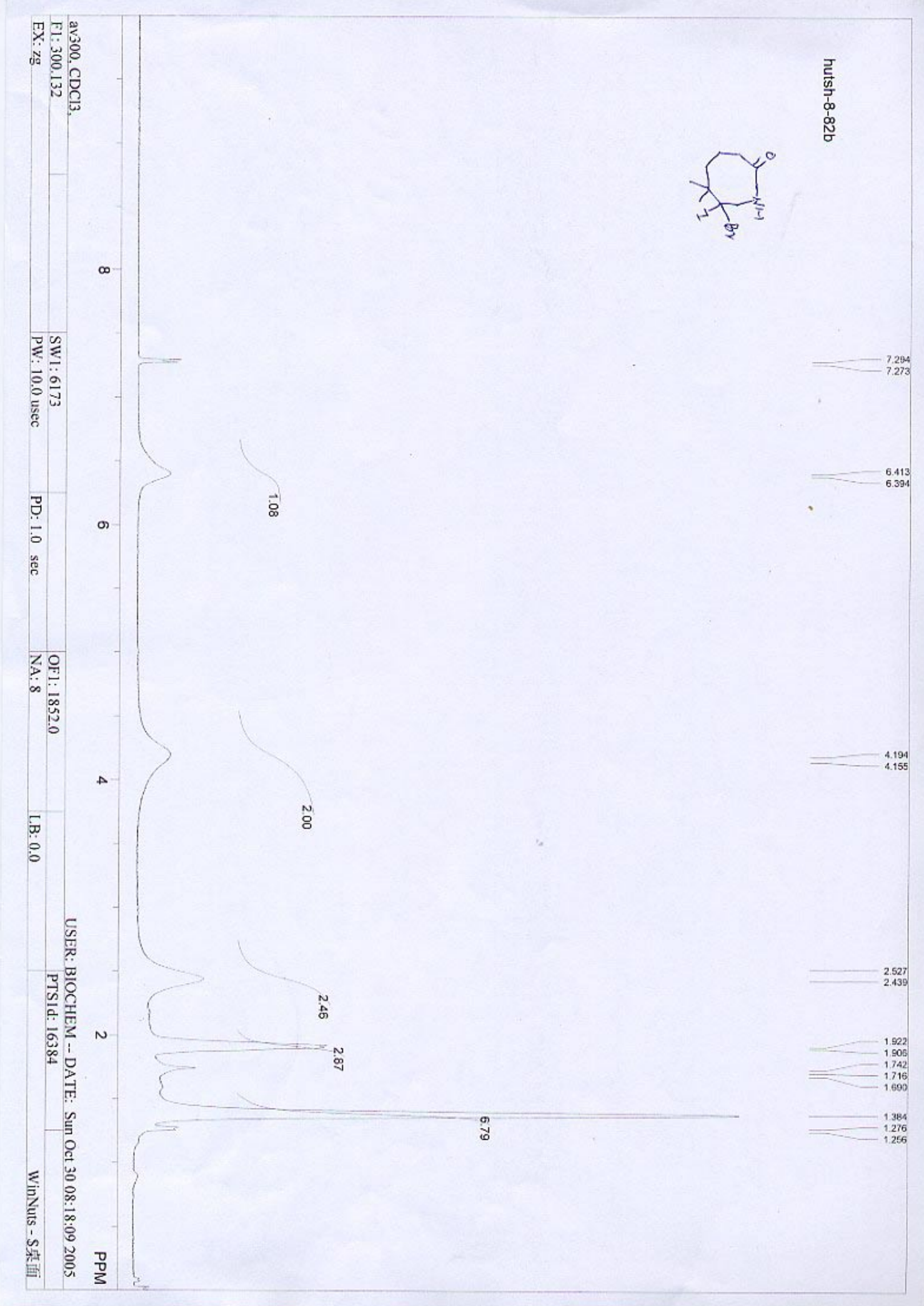


Compound 19c:

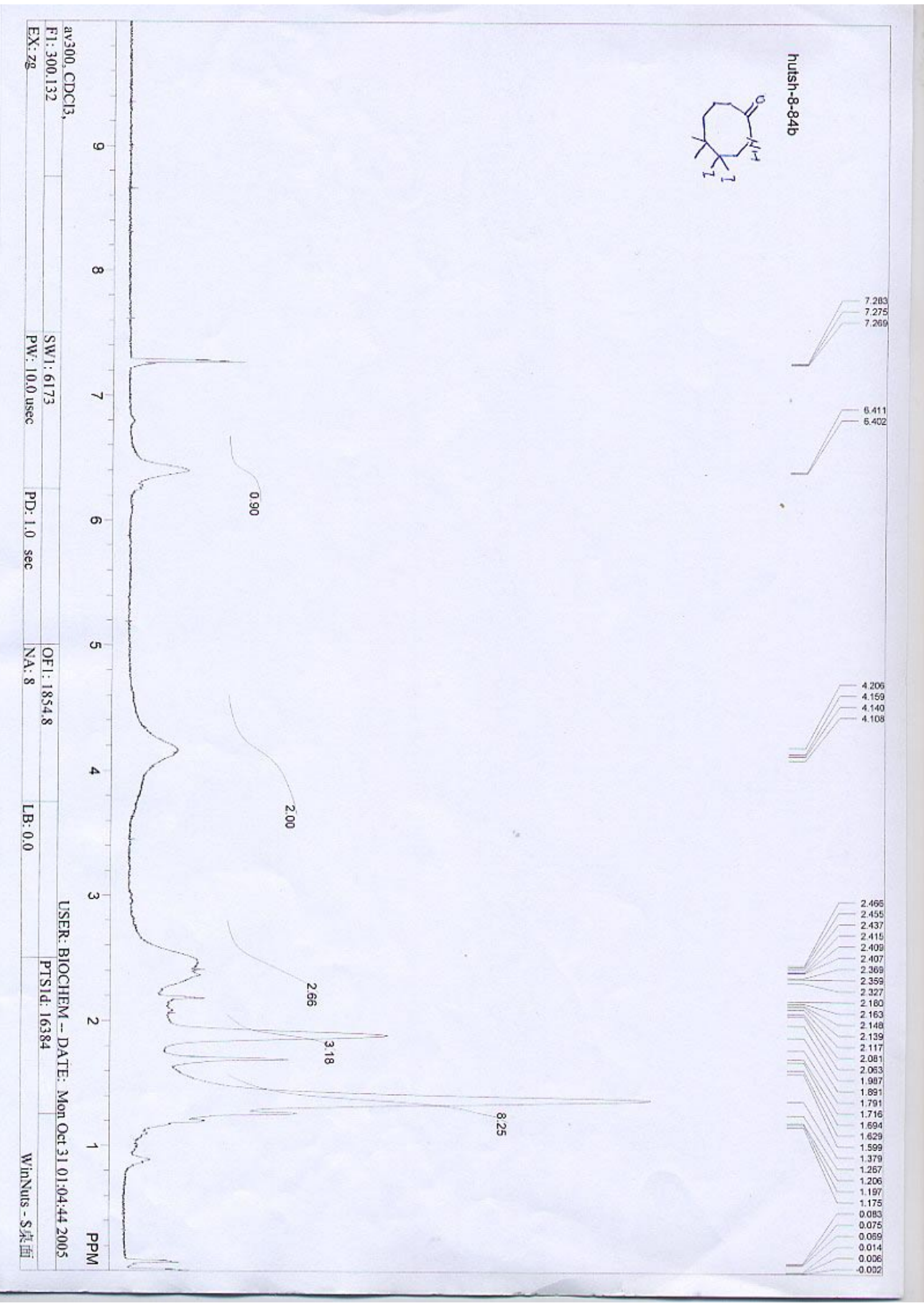


Compound 19f:

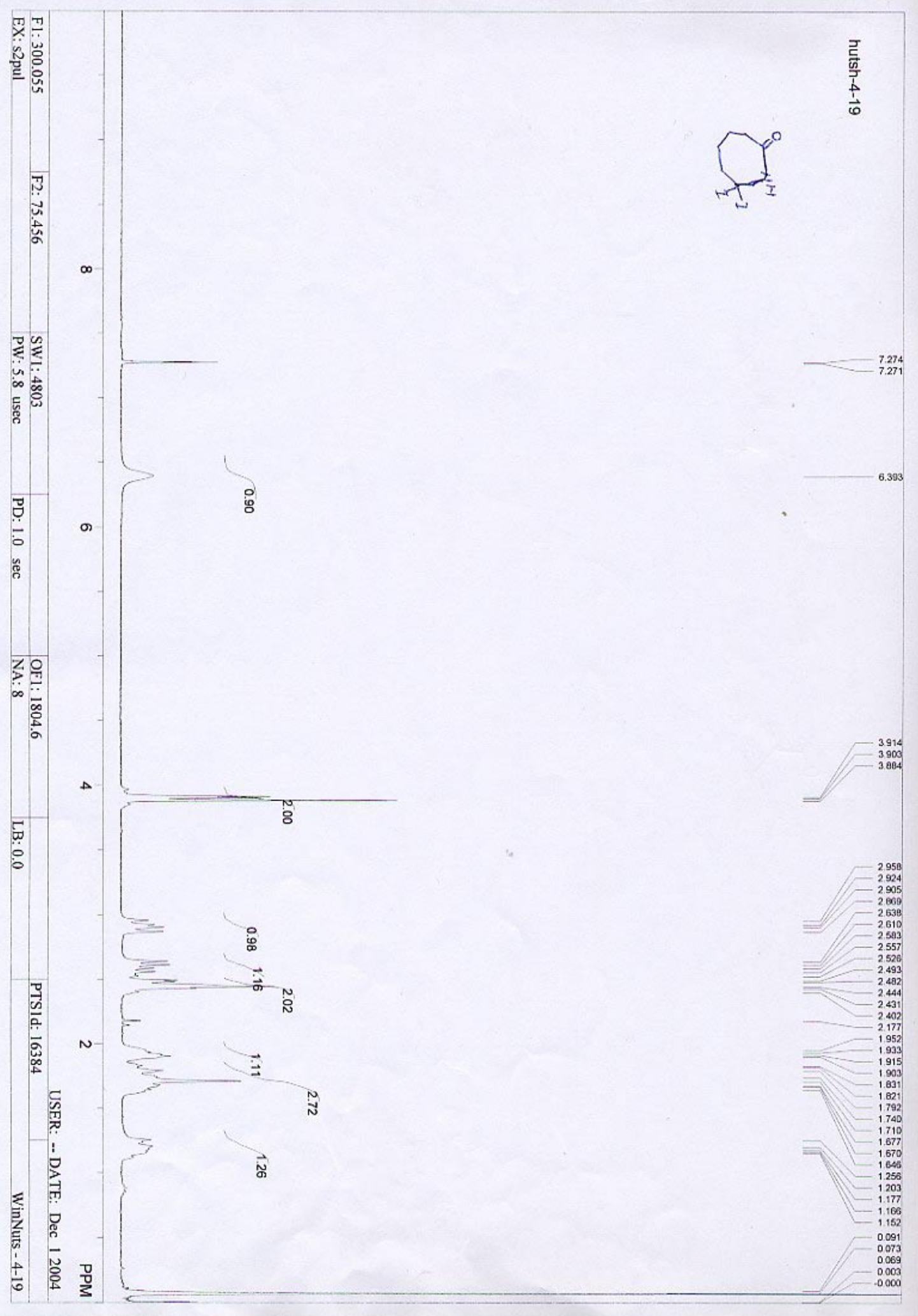


Compound 21a:

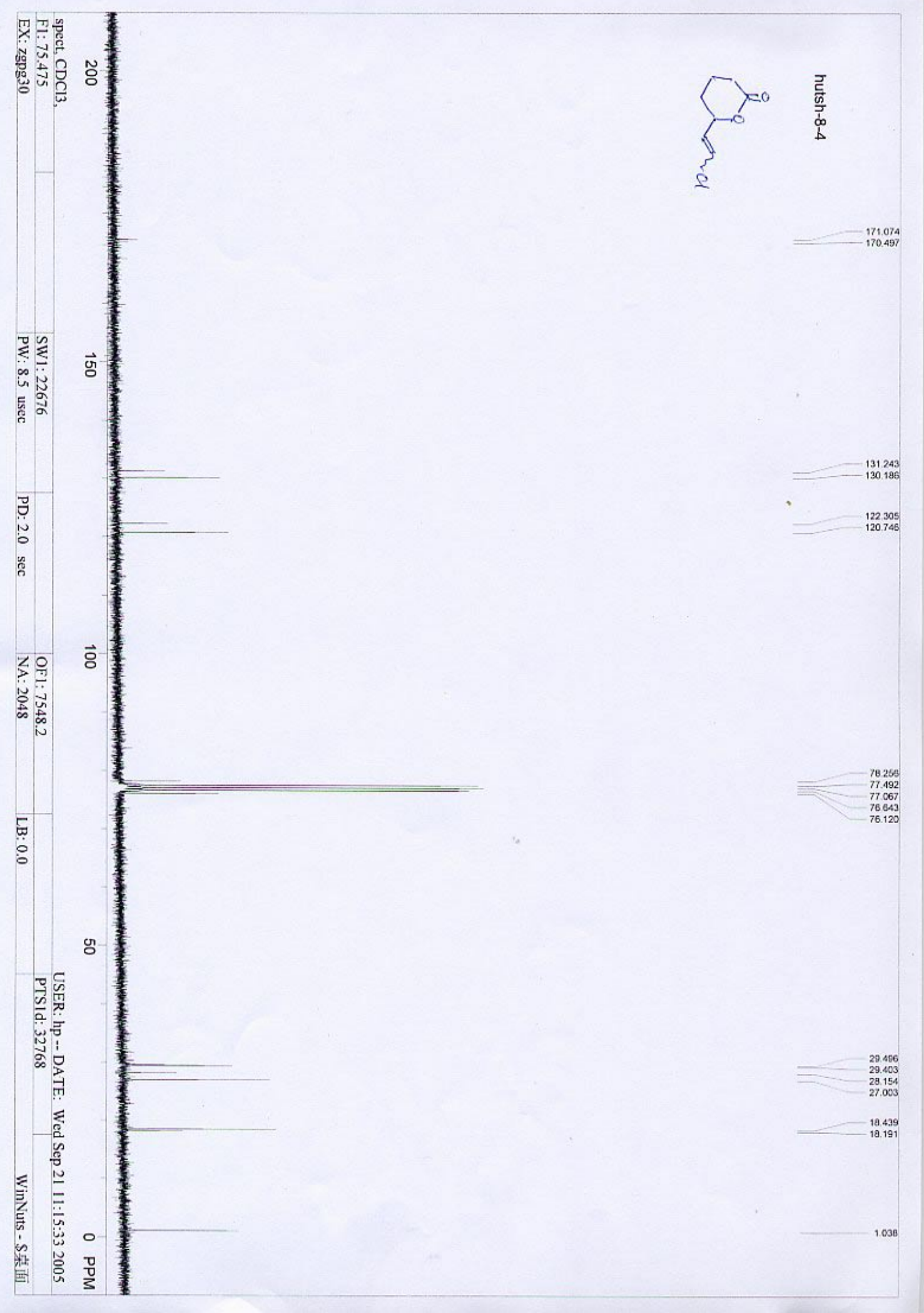


Compound 21c:

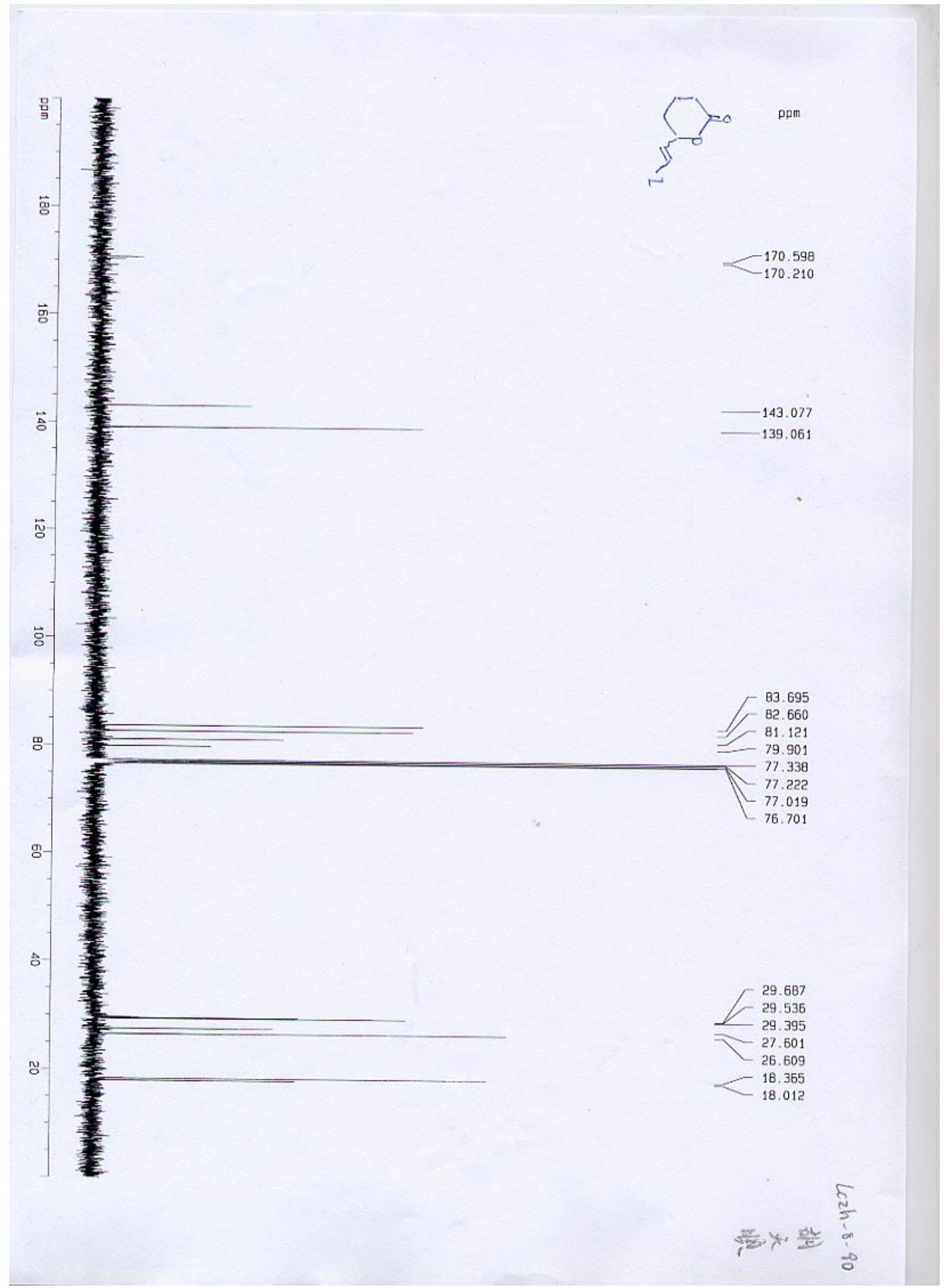

